\title{
1. GEOCHEMISTRY OF LEG 111 BASALTS: INTRUSIVE FEEDERS FOR HIGHLY DEPLETED PILLOWS AND FLOWS 1
}

\author{
L. K. Autio, J. W. Sparks, and J. M. Rhodes ${ }^{2}$
}

\begin{abstract}
Fifty samples of basalt recovered during ODP Leg 111 from the dikes (Layer 2C) of Hole 504B (1350.0-1562.3 m below seafloor) were analyzed by X-ray-fluorescence techniques. All of the samples are highly depleted in magmaphile elements relative to other mid-ocean ridge basalts, with $\mathrm{TiO}_{2}=0.75-1.24 \mathrm{wt} \%, \mathrm{Na}_{2} \mathrm{O}=1.59-2.22 \mathrm{wt} \%, \mathrm{Zr}=38-64$ $\mathrm{ppm}, \mathrm{Nb}=0.3-1.5 \mathrm{ppm}$, and $\mathrm{Y}=20-30 \mathrm{ppm}$ (for samples containing $0 \%-2 \%$ phenocrysts), but have ratios of highly incompatible elements similar to normal Type I mid-ocean ridge basalts (e.g., $\mathrm{Zr} / \mathrm{Nb}>30$ ). Abundances of compatible elements are similar to those of typical mid-ocean ridge basalts, with $\mathrm{MgO}=7.2-9.2 \mathrm{wt} \%, \mathrm{Fe}_{2} \mathrm{O}_{3}{ }^{*}=9.3-12.5 \mathrm{wt} \%$, $\mathrm{Ni}=55-164 \mathrm{ppm}$, and $\mathrm{Cr}=26-388 \mathrm{ppm}$. Approximately $2 \%$ of the samples recovered from the top part of Hole 504B are similar to normal Type I or Type II ocean floor basalts. However, all of the analyzed Leg 111 samples from Hole 504B are depleted basalts. Aphyric dike rocks from Leg 111 are virtually identical to the depleted aphyric samples recovered from the pillow lavas and dikes in the upper $1075 \mathrm{~m}$ of Hole 504B during DSDP Legs 69,70, and 83, with the exception of elements readily altered by seawater $(\mathrm{Sr}, \mathrm{Rb}$, and $\mathrm{K})$. These elements reach a maximum in both abundance and variability in the pillow lavas of the upper $571.5 \mathrm{~m}$ of Hole 504B and decline to more constant values in the dike system sampled on Legs 83 and 111, apparently as a result of a decrease in porosity and increase in alteration temperatures relative to the pillow lavas. Based on compositional similarities to the vast majority of the pillows and flows, the dikes sampled on Leg 111 appear to be the feeder system for the pillow lavas in the upper part of Hole 504B. The incompatible-element-depleted compositions of the Costa Rica Rift Zone basalts are consistent with multistage melting of a normal mid-ocean ridge source.
\end{abstract}

\section{INTRODUCTION}

The Costa Rica Rift Zone, at $3^{\circ} \mathrm{N}, 83^{\circ} \mathrm{W}$, between the Galapagos spreading center and the Malpelo Ridge (Shipboard Scientific Party, 1988), defines the junction of the Cocos and Nazca plates. During Legs $68,69,70$, and 83 of the Deep Sea Drilling Project (DSDP) a total of seven holes were drilled in old (3.95.9 m.y.) crust at two locations to the south of the modern spreading axis. Hole 504B was the major drill hole, located in 5.9-m.y.-old crust and penetrating $1075.5 \mathrm{~m}$ of basement. This was the deepest penetration of the oceanic crust at that time (Anderson et al., 1982) passing through $571.5 \mathrm{~m}$ of pillow basalts and massive flows (Layer 2A), $209 \mathrm{~m}$ of transition zone (Layer 2B), and $295 \mathrm{~m}$ of the sheeted dike complex (Layer 2C). Leg 111 of the Ocean Drilling Program (ODP) deepened Hole 504B another $212.3 \mathrm{~m}$ into the sheeted dike complex in an effort to reach the layered gabbroic complex (Layer 3), the presence of which was postulated by analogy to ophiolites (Christensen and Salisbury, 1975; Basaltic Volcanism Study Project, 1981).

A number of preliminary studies of igneous basalt compositions from the upper part of Hole 504B have been published on major element microprobe analyses of fresh glasses (Natland et al., 1983; Kempton, 1985), major and trace element whole-rock compositions (Autio and Rhodes, 1983; Marsh et al., 1983; Etoubleau et al., 1983; Sharaskin et al., 1983; Hubberton et al., 1983; Kempton et al., 1985; Emmermann, 1985; Tual et al., 1985), rare earth element analyses (Autio and Rhodes, 1983; Etoubleau et al., 1983; Sharaskin et al., 1983; Kempton et al., 1985; Tual et al., 1985; Emmermann, 1985), and isotopic analyses (Barrett and Friedrichsen, 1982; Barrett, 1983; Friedrichsen, 1985). Although all of these studies show an unusual depletion

\footnotetext{
${ }^{1}$ Becker, K., Sakai, H., et al., 1989. Proc. ODP, Sci. Results, 111: College Station, TX (Ocean Drilling Program).

2 Department of Geology and Geography, University of Massachusetts, Amherst, MA 01003 .
}

in incompatible elements $(\mathrm{Ti}, \mathrm{Zr}, \mathrm{Nb}, \mathrm{La}, \mathrm{Ce}$, etc.) occurring in $98 \%$ of the basalts relative to other mid-ocean ridge basalts, disagreement exists on the cause(s) of this depletion. Models range from postulation of the basalts as highly primitive (Natland et al., 1983; Shipboard Scientific Party, 1988) to derivation of the basalts by multistage melting followed by moderately extensive crystal fractionation (Autio and Rhodes, 1981, 1983; Autio, 1984; Kempton et al., 1985; Shipboard Scientific Party, 1988). In spite of the marked depletion in incompatible elements, the basalts exhibit isotopic and incompatible element ratios consistent with other Type I (as defined by Bryan et al., 1976) mid-ocean ridge basalts (Autio and Rhodes, 1983; Barrett and Friedrichsen, 1982). Additional work on the petrography (Natland et al., 1983; Kempton et al., 1985; Kempton, 1985) and experimental phase relations (Autio and Rhodes, 1983; Autio, 1984) of the Hole 504B basalts suggests that the magmas were close to multiple saturation with olivine plus plagioclase plus or minus clinopyroxene on the liquidus.

In order to evaluate the suitability of the intrusive (Layer 2C) magma compositions as feeders for the pillow lavas and flows (Layer 2A) of Hole 504B, 50 samples were chosen from the Leg 111 basalts for X-ray-fluorescence (XRF) analysis. These samples ranged from aphyric to moderately phyric $(0 \%-15 \%$ phenocrysts) and were carefully selected to minimize the effects of alteration. Both major $(\mathrm{Si}, \mathrm{Ti}, \mathrm{Al}, \mathrm{Fe}, \mathrm{Mn}, \mathrm{Mg}, \mathrm{Ca}, \mathrm{Na}, \mathrm{K}$, and $\mathrm{P})$ and trace (Rb, Sr, Y, Ga, Nb, Zr, Zn, Ni, Cr, and V) element abundances were determined for all samples. The nomenclature of Autio and Rhodes (1983) and Kempton et al. (1985) is followed for basalt types from the upper part of Hole 504B.

\section{ANALYTICAL TECHNIQUES}

Core samples of $10-30 \mathrm{~g}$ were carefully sawn and ground to remove alteration, hydrothermal veining, and saw marks and then crushed in a tungsten carbide shatterbox. Powders for major element XRF analysis were dried and oxidized at $1000^{\circ} \mathrm{C}$ in air for $2 \mathrm{hr}$. Samples for major element analysis were prepared as lanthanum-bearing lithium borate fusedglass disks (Norrish and Hutton, 1969). Concentrations were measured on an automated Siemens MRS 400MP 10-channel X-ray spectrograph, 
using an end-window rhodium tube. Trace elements were determined on an automated Siemens SRS-1 sequential X-ray spectrograph on unfired pressed-powder pellets. A molybdenum tube was used to measure $\mathrm{Y}, \mathrm{Sr}$, $\mathrm{Rb}$, and $\mathrm{Ga}$; a gold tube was used to measure $\mathrm{Nb}, \mathrm{Zr}, \mathrm{Zn}, \mathrm{Ni}, \mathrm{Cr}$, and $\mathrm{V}$. Corrections were made for background nonlinearity, interelement interference, and tube contamination using the methods developed by Norrish and Chappell (1967). Corrections for mass absorption coefficients were based on a modification of Reynolds' (1967) Compton scattering method. All samples were analyzed in duplicate.

The precision error in the Costa Rica Rift Zone basalt analyses (Table 1) is expressed as one standard deviation of the mean of duplicate determinations. The values were calculated by using the formula $s=$ $\sqrt{(\Delta / 2)^{2} /(n-1)}$, where $\Delta$ is the difference between duplicate analyses of the same element in a single sample and $n$ is the number of samples analyzed. Hence, the error terms refer to the precision of analyses at the concentration levels in the Costa Rica Rift Zone basalts under the given operating conditions. USGS basalt standard BHVO-1 was analyzed as an unknown with each run to provide an estimate of accuracy (Table 1). An interlaboratory standard from Leg 111 was also analyzed (Table 1).

$\mathrm{Mg}^{\prime}$-values were calculated from molar $\mathrm{MgO} /(\mathrm{MgO}+\mathrm{FeO})$, where $\mathrm{Fe}^{3+} /\left(\mathrm{Fe}^{3+}+\mathrm{Fe}^{2+}\right)$ is defined as 0.1 (Basaltic Volcanism Study Project, 1981). The terms $\mathrm{FeO}^{*}$ and $\mathrm{Fe}_{2} \mathrm{O}_{3}{ }^{*}$ refer respectively to total $\mathrm{Fe}$ expressed as weight percent $\mathrm{FeO}$ or $\mathrm{Fe}_{2} \mathrm{O}_{3}$. Chondrite-normalized abundances of rare earth elements are shown as $\mathrm{A}_{\mathrm{CH}}$ or $\mathrm{A} / \mathrm{B}_{\mathrm{CH}}$. Normalizing values are from F. A. Frey (pers. comm., 1982).

\section{RESULTS}

Throughout this paper, comparisons will be made to basalt compositions from the upper parts of Hole 504B and other mid-ocean ridge locations. Data for the upper part of Hole 504B are from Autio and Rhodes (1983) and Kempton et al. (1985). Data from a wide range of locations (DSDP Legs 2, 3, $9,17,34,45,49,52$, and 82 and ODP 106 and 109; dredged basalts from Kane Fracture Zone, Bouvet/Southwest Indian Ridge, Galapagos Rift Zone, Tamayo, Serocki, Cayman Trough, Juan de Fuca Ridge, Chain 21, 43, and 73, and FAMOUS) and various compilations of mid-ocean ridge basalt glass analyses were used to form a mid-ocean ridge basalt data base (Bass et al., 1973; Frey et al., 1974; Melson et al., 1976; Rhodes et al., 1976,

Table 1. Analytical precision of basalt analyses.

\begin{tabular}{|c|c|c|c|c|}
\hline & \multicolumn{2}{|c|}{$\begin{array}{c}\text { USGS basalt } \\
\text { standard BHVO-1 }\end{array}$} & \multirow{2}{*}{$\begin{array}{c}\text { Leg } 111 \\
\text { interlaboratory } \\
\text { standard }\end{array}$} & \multirow{2}{*}{$\begin{array}{c}\text { Costa Rica } \\
\text { Rift Zone } \\
\text { error }\end{array}$} \\
\hline & Average & $\begin{array}{l}\text { Standard } \\
\text { deviation }\end{array}$ & & \\
\hline \multicolumn{5}{|c|}{ Major elements (wt \%) } \\
\hline $\mathrm{SiO}_{2}$ & 49.75 & 0.04 & 50.01 & 0.09 \\
\hline $\mathrm{TiO}_{2}$ & 2.72 & 0.01 & 0.81 & 0.005 \\
\hline $\mathrm{Al}_{2} \mathrm{O}_{3}$ & 13.64 & 0.05 & 16.54 & 0.05 \\
\hline $\mathrm{Fe}_{2} \mathrm{O}_{3} *$ & 12.08 & 0.03 & 9.01 & 0.02 \\
\hline $\mathrm{MnO}$ & 0.17 & 0.00 & 0.16 & 0.001 \\
\hline $\mathrm{MgO}$ & 7.14 & 0.03 & 8.50 & 0.03 \\
\hline $\mathrm{CaO}$ & 11.32 & 0.01 & 13.31 & 0.03 \\
\hline $\mathrm{Na}_{2} \mathrm{O}$ & 2.24 & 0.06 & 1.68 & 0.03 \\
\hline $\mathrm{K}_{2} \mathrm{O}$ & 0.52 & 0.00 & 0.01 & 0.001 \\
\hline $\mathrm{P}_{2} \mathrm{O}_{5}$ & 0.27 & 0.00 & 0.05 & 0.002 \\
\hline \multicolumn{5}{|c|}{ Trace elements (ppm) } \\
\hline $\mathrm{Rb}$ & 9.1 & 0.3 & $<0.1$ & 0.3 \\
\hline $\mathrm{Sr}$ & 392 & 1 & 61.4 & 0.6 \\
\hline $\mathrm{Y}$ & 24.9 & 0.1 & 19.9 & 0.2 \\
\hline $\mathrm{Ga}$ & 21.1 & 0.3 & 16 & 1 \\
\hline $\mathrm{Nb}$ & 19.7 & 0.3 & 0.6 & 0.3 \\
\hline $\mathrm{Zr}$ & 185 & 0.9 & 40.3 & 0.5 \\
\hline $\mathrm{Zn}$ & 113 & 0.9 & 69 & 0.6 \\
\hline $\mathrm{Ni}$ & $a_{133}$ & 2 & 128 & 0.8 \\
\hline $\mathrm{Cr}$ & 294 & 2 & 374 & 2 \\
\hline V & 292 & 3 & 227 & 3 \\
\hline
\end{tabular}

a This pellet was later found to be contaminated with nickel. Uncontaminated powders have values of $123 \pm 2 \mathrm{ppm} \mathrm{Ni}$.
1978; Ballard et al., 1979; Tarney et al., 1979; Byerly and Sinton, 1979: Schilling et al., 1983; Fisk et al., 1982; LeRoex et al., 1983; Bender et al., 1984; Drake et al., 1985; Humphris et al., in press; J. M. Rhodes, unpubl. data; R. A. Liias, unpubl. data; W. B. Bryan, unpubl. data).

Compositions of the Leg 111 basaltic dike samples are given in Table 2. Concentration ranges and averages are given in Table 3 for all samples and for those samples containing less than $2 \%$ phenocrysts. Ranges for aphyric to sparsely phyric samples are nearly identical to ranges for all samples, and the averages differ only slightly. The Leg 111 basalts exhibit moderate concentrations of compatible elements (e.g., $\mathrm{MgO}=7.2-9.2 \mathrm{wt} \%, \mathrm{Fe}_{2} \mathrm{O}_{3}{ }^{*}$ $=9.3-12.5 \mathrm{wt} \%, \mathrm{Ni}=55-164 \mathrm{ppm}$, and $\mathrm{Cr}=26-413 \mathrm{ppm})$. $\mathrm{Mg}^{\prime}$-values range from 0.56 to 0.69 . Moderately incompatible elements are depleted, with $\mathrm{TiO}_{2}=0.75-1.24 \mathrm{wt} \%, \mathrm{Na}_{2} \mathrm{O}=$ $1.6-2.5 \mathrm{wt} \%, \mathrm{Y}=20-30 \mathrm{ppm}$, and $\mathrm{Sr}=44-67 \mathrm{ppm}$. The abundances of highly incompatible elements are lower than those of most other mid-ocean ridge basalts, with $\mathrm{K}_{2} \mathrm{O}=0.01-0.02$ wt \%, $\mathrm{P}_{2} \mathrm{O}_{5}=0.05-0.08 \mathrm{wt} \%, \mathrm{Rb}<0.5 \mathrm{ppm}, \mathrm{Zr}=38-64$ $\mathrm{ppm}$, and $\mathrm{Nb}=0.2-1.6 \mathrm{ppm}$. These abundances are similar to those of the Group D (depleted) basalts from the upper part of Hole 504B (Autio and Rhodes, 1983; Kempton et al., 1985).

Average analyses of aphyric to sparsely phyric Group D basalts $(0 \%-2 \%$ phenocrysts) from Legs $69,70,83$, and 111 exhibit no significant differences in immobile major and trace element abundances (Table 3). Apparent correlations of abundances of these elements with depth (Shipboard Scientific Party, 1988) result from the inclusion of variably phyric samples. Concentrations of mobile major and trace elements do, however, differ from the top to the bottom of Hole 504B (Table 3). K, Rb, and $\mathrm{Sr}$ decrease with increasing depth (Figs. 1-3), reaching their maximum values and variability in the pillow lavas of Legs 69 , 70 , and $83(0.14 \pm 0.09 \mathrm{wt} \%, 2.2 \pm 2.0 \mathrm{ppm}$, and $66.9 \pm$ $6.0 \mathrm{ppm}$, respectively), and abruptly decreasing to more constant values in the less porous transition ( Leg 83: $\mathrm{K}=0.02 \pm$ $0.01 \mathrm{wt} \%, \mathrm{Rb}=0.5 \pm 0.1 \mathrm{ppm}$, and $\mathrm{Sr}=53.4 \pm 6.1 \mathrm{ppm})$ and dike zones (Legs 83 and $111: \mathrm{K}=0.01 \pm<0.01 \mathrm{wt} \%, \mathrm{Rb}$ $=0.1 \pm 0.1 \mathrm{ppm}$, and $\mathrm{Sr}=57.9 \pm 6.4 \mathrm{ppm})$. Honnorez et al. (1983) and Alt et al. (1986) have discussed the alteration chemistry of the top of Hole 504B in detail. Other elements, such as $\mathrm{Zr}$, Y, and Ti (Fig. 4), do not vary with depth or show correlations to the more easily altered elements and are thus presumed to be close to their abundances at the time of crystallization.

Chemical units were determined on the basis of jumps in concentrations of immobile incompatible major and trace elements, particularly $\mathrm{Ti}, \mathrm{Zr}, \mathrm{Nb}$, and $\mathrm{Y}$ (Table 2). Changes in concentration of more than $10 \%$ were presumed to be the result of new influxes of magma, rather than variations caused by fractionation within a single flow or analytical error. The chemical units roughly parallel the units determined by hand-sample and petrographic examination aboard ship (Shipboard Scientific Party, 1988).

The Leg 111 basalts have $\mathrm{Mg}^{\prime}$-values $(0.56-0.69)$ that are identical to those of the aphyric samples from the upper part of Hole 504B (Fig. 5) and similar to values of other moderately evolved mid-ocean ridge basalts. Sample 111-504B-159R-1, 52$56 \mathrm{~cm}$ (Piece 9), is the most evolved Group D sample from this location, with an $\mathrm{Mg}^{\prime}$-value of 0.56 . Similarly, a plot of $\mathrm{Ni}$ vs. $\mathrm{Zr}$ (Fig. 6) shows $\mathrm{Ni}$ abundances identical to those of aphyric samples from the top of Hole 504B and comparable to abundances of other moderately evolved mid-ocean ridge basalts. Relative to other mid-ocean ridge basalts, however, $\mathrm{Zr}$ abundances are much lower than normal for a given $\mathrm{Ni}$ concentration. Fractionation paths calculated from experimental data (Autio, 1984) for a normal primitive mid-ocean ridge basalt from Chain 43 on the Mid-Atlantic Ridge and for one of the least evolved Costa Rica Rift Zone basalts are shown in Figure 6. Both sam- 
Table 2. Analyses of Hole 504B samples, Leg 111.

\begin{tabular}{|c|c|c|c|c|c|c|c|c|c|c|c|c|c|c|c|c|c|}
\hline $\begin{array}{l}\text { Group }{ }^{\mathrm{a}} \\
\text { Phyric }^{\mathrm{b}} \\
\text { Depth (mbsf) } \\
\text { Unit }^{\mathrm{c}}\end{array}$ & $\begin{array}{c}142 \mathrm{R}-2, \\
39-4 \mathrm{~cm} \\
\text { (Piece 6) } \\
1 \\
\mathrm{M} \\
1354.69 \\
153\end{array}$ & $\begin{array}{c}143 R-1 \\
81-86 \mathrm{~cm} \\
\text { (Piece 13) } \\
2 \\
\mathrm{M} \\
1360.11 \\
155\end{array}$ & $\begin{array}{c}\text { 143R-1, } \\
\text { 105-107 cm } \\
\text { (Piece 17) } \\
2 \\
\text { S } \\
1360.35 \\
155\end{array}$ & $\begin{array}{c}\text { 143R-1, } \\
113-115 \mathrm{~cm} \\
\text { (Piece 18) } \\
3 \\
\text { A } \\
1360.43 \\
155\end{array}$ & $\begin{array}{c}143 \mathrm{R}-2, \\
34-36 \mathrm{~cm} \\
\text { (Piece 6) } \\
4 \\
\mathrm{~S} \\
1361.14 \\
155\end{array}$ & $\begin{array}{c}144 \mathrm{R}-1, \\
22-2 \mathrm{~cm} \\
\text { (Piece 4) } \\
4 \\
\text { S } \\
1368.92 \\
157\end{array}$ & $\begin{array}{c}144 \mathrm{R}-1, \\
49-52 \mathrm{~cm} \\
\text { (Piece 8) } \\
4 \\
\text { S } \\
1369.19 \\
157\end{array}$ & $\begin{array}{c}\text { 144R-1, } \\
96-99 \mathrm{~cm} \\
\text { (Piece 14) } \\
5 \\
\text { A } \\
1369.66 \\
159\end{array}$ & $\begin{array}{c}\text { 145R-1, } \\
80-84 \mathrm{~cm} \\
\text { (Piece 10) } \\
6 \\
\mathrm{~S} \\
1379.10 \\
160\end{array}$ & $\begin{array}{c}145 \mathrm{R}-2, \\
7-9 \mathrm{~cm} \\
\text { (Piece 2) } \\
6 \\
\text { S } \\
1379.87 \\
160\end{array}$ & $\begin{array}{c}145 \mathrm{R}-2, \\
115-119 \mathrm{~cm} \\
\text { (Piece 10) } \\
6 \\
\mathrm{P} \\
1380.95 \\
160\end{array}$ & $\begin{array}{c}145 \mathrm{~S}-3, \\
32-34 \mathrm{~cm} \\
\text { (Piece } 3 \text { ) } \\
6 \\
\mathrm{M} \\
1381.62 \\
160\end{array}$ & $\begin{array}{c}\text { 145R-3, } \\
88-91 \mathrm{~cm} \\
\text { (Piece 6) } \\
6 \\
\text { P } \\
1382.18 \\
160\end{array}$ & $\begin{array}{c}145 \mathrm{R}-4, \\
15-17 \mathrm{~cm} \\
\text { (Piece 3) } \\
7 \\
\mathrm{~S} \\
1382.95 \\
161\end{array}$ & $\begin{array}{c}147 \mathrm{R}-1, \\
23-28 \mathrm{~cm} \\
\text { (Piece 5) } \\
8 \\
\text { S } \\
1397.63 \\
163\end{array}$ & $\begin{array}{c}\text { 147R-1, } \\
66-68 \mathrm{~cm} \\
\text { (Piece 8) } \\
8 \\
\mathrm{~S} \\
1398.06 \\
163\end{array}$ & $\begin{array}{c}147 \mathrm{R}-2, \\
4-6 \mathrm{~cm} \\
\text { (Piece 1) } \\
8 \\
\mathrm{~S} \\
1398.94 \\
163\end{array}$ \\
\hline \multicolumn{18}{|c|}{ Major elements (wt\%) } \\
\hline $\begin{array}{l}\mathrm{SiO}_{2} \\
\mathrm{TiO}_{2} \\
\mathrm{Al}_{2} \mathrm{O}_{3} \\
\mathrm{Fe}_{2} \mathrm{O}_{3} \\
\mathrm{MnO} \\
\mathrm{MgO} \\
\mathrm{CaO} \\
\mathrm{Na}{ }_{2} \mathrm{O} \\
\mathrm{K}_{2} \mathrm{O} \\
\mathrm{P}_{2} \mathrm{O}_{5}\end{array}$ & $\begin{array}{c}50.4 \\
0.94 \\
15.62 \\
10.26 \\
0.17 \\
7.84 \\
12.80 \\
1.96 \\
0.01 \\
0.06\end{array}$ & $\begin{array}{r}49.1 \\
0.84 \\
16.52 \\
9.69 \\
0.16 \\
8.84 \\
13.30 \\
1.77 \\
0.01 \\
0.05\end{array}$ & $\begin{array}{r}49.3 \\
0.81 \\
16.69 \\
9.44 \\
0.16 \\
8.93 \\
13.09 \\
1.92 \\
0.01 \\
0.05\end{array}$ & $\begin{array}{r}48.9 \\
0.75 \\
16.90 \\
9.26 \\
0.15 \\
9.18 \\
13.16 \\
1.72 \\
0.01 \\
0.05\end{array}$ & $\begin{array}{r}49.5 \\
0.81 \\
16.35 \\
9.50 \\
0.16 \\
8.95 \\
13.17 \\
1.80 \\
0.01 \\
0.05\end{array}$ & $\begin{array}{r}49.5 \\
0.82 \\
16.42 \\
9.70 \\
0.17 \\
9.02 \\
13.07 \\
1.76 \\
0.02 \\
0.05\end{array}$ & $\begin{array}{c}49.1 \\
0.83 \\
16.84 \\
9.65 \\
0.16 \\
8.62 \\
13.16 \\
1.90 \\
0.01 \\
0.05\end{array}$ & $\begin{array}{r}50.9 \\
1.06 \\
14.38 \\
11.34 \\
0.19 \\
7.99 \\
12.36 \\
1.96 \\
0.02 \\
0.07\end{array}$ & $\begin{array}{r}50.1 \\
0.87 \\
15.57 \\
9.67 \\
0.16 \\
8.96 \\
13.09 \\
1.63 \\
0.01 \\
0.06\end{array}$ & $\begin{array}{c}50.1 \\
0.86 \\
15.94 \\
9.74 \\
0.16 \\
8.32 \\
13.06 \\
1.79 \\
0.01 \\
0.05\end{array}$ & $\begin{array}{r}50.4 \\
0.88 \\
15.94 \\
9.58 \\
0.16 \\
8.30 \\
13.16 \\
1.81 \\
0.01 \\
0.06\end{array}$ & $\begin{array}{r}50.3 \\
0.87 \\
15.80 \\
9.64 \\
0.15 \\
8.68 \\
13.10 \\
1.78 \\
0.01 \\
0.06\end{array}$ & $\begin{array}{c}50.1 \\
0.86 \\
15.48 \\
9.77 \\
0.16 \\
9.04 \\
12.99 \\
1.71 \\
0.01 \\
0.06\end{array}$ & $\begin{array}{c}50.8 \\
0.96 \\
15.20 \\
10.56 \\
0.17 \\
8.02 \\
12.77 \\
1.77 \\
0.01 \\
0.06\end{array}$ & $\begin{array}{c}50.4 \\
0.88 \\
16.02 \\
9.35 \\
0.16 \\
8.30 \\
13.05 \\
1.95 \\
0.01 \\
0.06\end{array}$ & $\begin{array}{r}50.3 \\
0.89 \\
15.74 \\
9.46 \\
0.16 \\
8.34 \\
13.12 \\
1.94 \\
0.01 \\
0.06\end{array}$ & $\begin{array}{r}50.6 \\
0.89 \\
15.82 \\
9.39 \\
0.16 \\
8.35 \\
13.06 \\
2.02 \\
0.01 \\
0.06\end{array}$ \\
\hline & $\overline{100.06}$ & $\overline{100.28}$ & $\overline{100.40}$ & $\overline{100.08}$ & $\overline{100.30}$ & $\overline{100.53}$ & $\overline{100.32}$ & $\overline{100.27}$ & $\overline{100.12}$ & $\overline{100.03}$ & $\overline{100.30}$ & $\overline{100.39}$ & $\overline{100.18}$ & $\overline{100.32}$ & $\overline{100.18}$ & $\overline{100.02}$ & $\overline{100.36}$ \\
\hline \multicolumn{18}{|c|}{ Trace elements (ppm) } \\
\hline $\begin{array}{l}\mathrm{Rb} \\
\mathrm{Sr}\end{array}$ & $\begin{array}{r}0.1 \\
51.7\end{array}$ & $\begin{array}{r}0.1 \\
59.8\end{array}$ & $\begin{array}{r}0.0 \\
60.0\end{array}$ & $\begin{array}{r}0.2 \\
58.4\end{array}$ & $\begin{array}{r}0.2 \\
58.7\end{array}$ & $\begin{array}{r}0.3 \\
59.6\end{array}$ & $\begin{array}{r}0.0 \\
61.9\end{array}$ & $\begin{array}{r}0.3 \\
43.7\end{array}$ & $\begin{array}{r}0.1 \\
54.6\end{array}$ & $\begin{array}{r}0.4 \\
56.1\end{array}$ & $\begin{array}{r}0.0 \\
57.0\end{array}$ & $\begin{array}{r}0.2 \\
56.4\end{array}$ & $\begin{array}{r}0.0 \\
54.6\end{array}$ & $\begin{array}{r}0.0 \\
44.9\end{array}$ & $\begin{array}{r}0.0 \\
64.0\end{array}$ & $\begin{array}{r}0.2 \\
63.4\end{array}$ & $\begin{array}{r}0.0 \\
65.0\end{array}$ \\
\hline $\begin{array}{l}\text { Y } \\
\text { Ga }\end{array}$ & $\begin{array}{l}24.2 \\
16\end{array}$ & $\begin{array}{l}22.3 \\
14\end{array}$ & $\begin{array}{l}21.5 \\
15\end{array}$ & $\begin{array}{l}20.1 \\
15\end{array}$ & $\begin{array}{l}21.7 \\
14\end{array}$ & $\begin{array}{l}22.3 \\
14\end{array}$ & $\begin{array}{l}22.6 \\
15\end{array}$ & $\begin{array}{l}28.3 \\
16\end{array}$ & $\begin{array}{l}21.8 \\
16\end{array}$ & $\begin{array}{l}\begin{array}{l}21.5 \\
16\end{array} \\
16\end{array}$ & $\begin{array}{l}22.5 \\
16\end{array}$ & $\begin{array}{l}21.6 \\
15\end{array}$ & $\begin{array}{l}\begin{array}{l}21.4 \\
15\end{array} \\
15\end{array}$ & $\begin{array}{l}25.6 \\
16\end{array}$ & $\begin{array}{l}21.1 \\
16\end{array}$ & $\begin{array}{l}21.7 \\
15\end{array}$ & $\begin{array}{l}22.2 \\
16\end{array}$ \\
\hline $\mathrm{Nb}$ & 1.0 & 0.8 & 0.5 & 0.9 & 0.7 & 1.0 & 0.4 & 0.3 & 0.8 & 0.5 & 0.5 & 1.2 & 0.4 & 0.7 & 0.5 & 1.2 & 0.9 \\
\hline $\mathrm{Zr}$ & 46.4 & 43.4 & 41.8 & 38.4 & 42.8 & 42.6 & 43.7 & 51.1 & 43.1 & 42.2 & 44.0 & 42.4 & 40.8 & 45.8 & 43.8 & 44.9 & 44.6 \\
\hline $\mathrm{Zn}$ & 73.1 & 83.8 & 75.5 & 80.4 & 91.8 & 69.0 & 86.1 & 98.8 & 58.6 & 55.3 & 54.7 & 56.4 & 57.0 & 78.2 & 63.5 & 64.6 & 66.6 \\
\hline $\mathrm{Ni}$ & 95 & 148 & 148 & 164 & 144 & 154 & 147 & 94 & 134 & 110 & 107 & 119 & 137 & 95 & 112 & 111 & 110 \\
\hline $\mathrm{Cr}$ & 197 & 358 & 333 & 358 & 354 & 358 & 359 & 212 & 388 & 365 & 369 & 376 & 413 & 243 & 346 & 367 & 361 \\
\hline $\mathrm{v}$ & 271 & 226 & 219 & 213 & 228 & 225 & 232 & 345 & 237 & 246 & 243 & 244 & 237 & 300 & 234 & 233 & 241 \\
\hline
\end{tabular}

Refers to chemical units (see text).

$\mathrm{A}=$ aphyric $(0 \%$ phenocrysts); $\mathrm{S}=$ sparsely phyric $(0 \%-2 \%$ phenocrysts); $\mathrm{M}=$ moderately phyric $(3 \%-5 \%$ phenocrysts); $\mathrm{P}=$ phyric $(>5 \%$ phenocrysts).

${ }^{\mathcal{C}}$ Flow units determined by petrographic and hand-sample methods (Shipboard Scientific Party, 1988). 
Table 2 (continued).

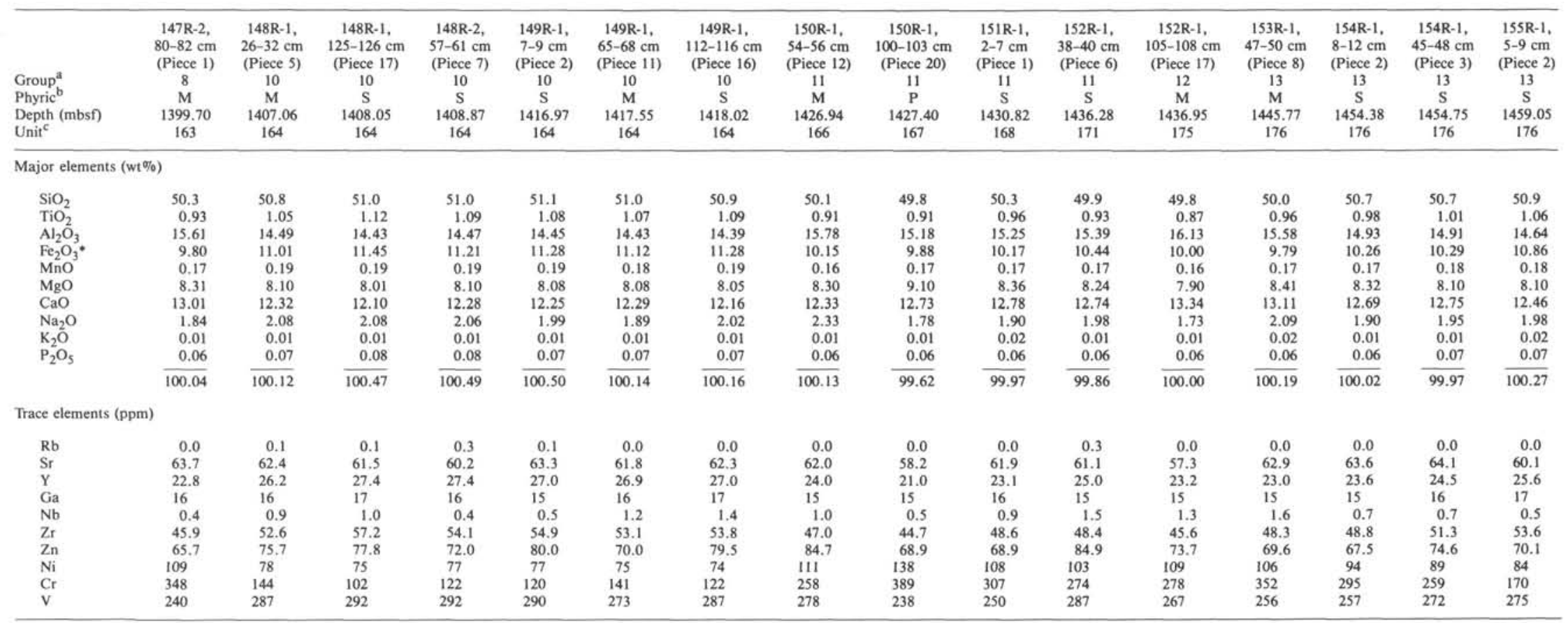


Table 2 (continued).

\begin{tabular}{|c|c|c|c|c|c|c|c|c|c|c|c|c|c|c|c|c|c|}
\hline $\begin{array}{l}\text { Group }^{\mathrm{a}} \\
\text { Phyric }^{\mathrm{b}} \\
\text { Depth (mbsf) } \\
\text { Unit }^{\mathrm{c}}\end{array}$ & $\begin{array}{c}156 \mathrm{R}-1, \\
19-20 \mathrm{~cm} \\
\text { (Piece 4) } \\
13 \\
\text { A } \\
1463.99 \\
177\end{array}$ & $\begin{array}{c}157 \mathrm{R}-1, \\
44-48 \mathrm{~cm} \\
\text { (Piece 8) } \\
14 \\
\text { S } \\
1473.54 \\
181\end{array}$ & $\begin{array}{c}158 \mathrm{R}-1, \\
42-46 \mathrm{~cm} \\
\text { (Piece 6) } \\
14 \\
\mathrm{~S} \\
1482.92 \\
182\end{array}$ & $\begin{array}{c}159 \mathrm{R}-1, \\
20-22 \mathrm{~cm} \\
\text { (Piece 4) } \\
15 \\
\mathrm{~S} \\
1488.30 \\
183\end{array}$ & $\begin{array}{c}159 \mathrm{R}-1, \\
52-56 \mathrm{~cm} \\
\text { (Piece 9) } \\
16 \\
\text { S } \\
1488.62 \\
184\end{array}$ & $\begin{array}{c}160 \mathrm{R}-1, \\
18-21 \mathrm{~cm} \\
\text { (Piece 4) } \\
17 \\
\mathrm{~S} \\
1494.88 \\
185\end{array}$ & $\begin{array}{c}161 \mathrm{R}-1, \\
23-27 \mathrm{~cm} \\
\text { (Piece 3) } \\
18 \\
\mathrm{M} \\
1504.33 \\
187\end{array}$ & $\begin{array}{c}161 \mathrm{R}-1, \\
53-55 \mathrm{~cm} \\
\text { (Piece 10) } \\
18 \\
\text { P } \\
1504.63 \\
187\end{array}$ & $\begin{array}{c}163 \mathrm{R}-1, \\
29-34 \mathrm{~cm} \\
\text { (Piece } 7 \text { ) } \\
19 \\
\mathrm{M} \\
1511.89 \\
187\end{array}$ & $\begin{array}{c}163 \mathrm{R}-1, \\
73-75 \mathrm{~cm} \\
\text { (Piece 13) } \\
19 \\
\mathrm{~S} \\
1512.33 \\
187\end{array}$ & $\begin{array}{c}163 \mathrm{R}-1, \\
138-142 \mathrm{~cm} \\
\text { (Piece 27) } \\
20 \\
\mathrm{~S} \\
1512.98 \\
187\end{array}$ & $\begin{array}{c}163 \mathrm{R}-2, \\
49-54 \mathrm{~cm} \\
\text { (Piece 7) } \\
21 \\
\mathrm{M} \\
1513.59 \\
188\end{array}$ & $\begin{array}{c}164 \mathrm{R}-1, \\
37-41 \mathrm{~cm} \\
\text { (Piece 6) } \\
21 \\
\mathrm{M} \\
1515.47 \\
188\end{array}$ & $\begin{array}{c}165 \mathrm{R}-1, \\
48-53 \mathrm{~cm} \\
\text { (Piece 6) } \\
21 \\
\mathrm{M} \\
1529.98 \\
189\end{array}$ & $\begin{array}{c}169 \mathrm{R}-1, \\
24-27 \mathrm{~cm} \\
\text { (Piece 6) } \\
22 \\
\mathbf{S} \\
1547.84 \\
191\end{array}$ & $\begin{array}{c}169 \mathrm{R}-1, \\
87-89 \mathrm{~cm} \\
\text { (Piece 17) } \\
22 \\
\mathrm{~S} \\
1548.47 \\
191\end{array}$ & $\begin{array}{c}170 \mathrm{R}-1, \\
27-31 \mathrm{~cm}, \\
\text { (Piece 6) } \\
22 \\
\mathrm{M} \\
1553.87 \\
191\end{array}$ \\
\hline \multicolumn{18}{|c|}{ Major elements ( $w t \%)$} \\
\hline $\begin{array}{l}\mathrm{SiO}_{2} \\
\mathrm{TiO}_{2} \\
\mathrm{Al}_{2} \mathrm{O}_{3} \\
\mathrm{Fe}_{2} \mathrm{O}_{3} \\
\mathrm{MnO} \\
\mathrm{MgO} \\
\mathrm{CaO} \\
\mathrm{Na}_{2} \mathrm{O} \\
\mathrm{K}_{2} \mathrm{O} \\
\mathrm{P}_{2} \mathrm{O}_{5}\end{array}$ & $\begin{array}{r}50.4 \\
1.01 \\
14.54 \\
11.24 \\
0.19 \\
8.06 \\
12.72 \\
1.67 \\
0.02 \\
0.07 \\
\end{array}$ & $\begin{array}{c}50.1 \\
0.95 \\
14.78 \\
11.02 \\
0.20 \\
8.51 \\
12.64 \\
2.00 \\
0.02 \\
0.06 \\
\end{array}$ & $\begin{array}{r}49.8 \\
0.96 \\
14.91 \\
10.87 \\
0.18 \\
8.21 \\
12.70 \\
2.16 \\
0.02 \\
0.06 \\
\end{array}$ & $\begin{array}{r}50.8 \\
1.12 \\
14.33 \\
11.43 \\
0.19 \\
7.94 \\
12.22 \\
2.02 \\
0.01 \\
0.07 \\
\end{array}$ & $\begin{array}{r}50.7 \\
1.24 \\
13.80 \\
12.50 \\
0.21 \\
7.16 \\
11.70 \\
2.22 \\
0.01 \\
0.08 \\
\end{array}$ & $\begin{array}{c}50.7 \\
1.07 \\
14.49 \\
11.09 \\
0.19 \\
8.18 \\
12.38 \\
1.93 \\
0.02 \\
0.07 \\
\end{array}$ & $\begin{array}{c}50.3 \\
0.91 \\
15.51 \\
10.45 \\
0.16 \\
8.01 \\
12.85 \\
1.87 \\
0.02 \\
0.06 \\
\end{array}$ & $\begin{array}{r}50.7 \\
0.91 \\
15.71 \\
10.26 \\
0.16 \\
7.81 \\
12.97 \\
1.70 \\
0.01 \\
0.06 \\
\end{array}$ & $\begin{array}{r}50.5 \\
1.03 \\
15.88 \\
10.21 \\
0.15 \\
7.61 \\
12.75 \\
1.98 \\
0.02 \\
0.07 \\
\end{array}$ & $\begin{array}{r}49.9 \\
1.07 \\
15.95 \\
10.46 \\
0.16 \\
7.88 \\
12.46 \\
2.42 \\
0.01 \\
0.07 \\
\end{array}$ & $\begin{array}{c}50.0 \\
0.97 \\
16.79 \\
9.84 \\
0.15 \\
7.59 \\
12.50 \\
2.51 \\
0.02 \\
0.06 \\
\end{array}$ & $\begin{array}{r}49.9 \\
1.03 \\
16.43 \\
10.11 \\
0.16 \\
7.65 \\
12.88 \\
2.26 \\
0.01 \\
0.07 \\
\end{array}$ & $\begin{array}{c}50.3 \\
0.98 \\
15.14 \\
10.68 \\
0.17 \\
8.36 \\
12.47 \\
1.78 \\
0.02 \\
0.07 \\
\end{array}$ & $\begin{array}{r}50.9 \\
1.01 \\
15.35 \\
10.64 \\
0.18 \\
7.84 \\
12.45 \\
1.79 \\
0.01 \\
0.07 \\
\end{array}$ & $\begin{array}{r}50.3 \\
0.92 \\
15.14 \\
10.38 \\
0.17 \\
8.47 \\
13.06 \\
1.59 \\
0.01 \\
0.06 \\
\end{array}$ & $\begin{array}{c}50.4 \\
0.92 \\
15.23 \\
10.35 \\
0.17 \\
8.51 \\
13.12 \\
1.61 \\
0.01 \\
0.06 \\
\end{array}$ & $\begin{array}{c}50.1 \\
0.91 \\
15.16 \\
10.39 \\
0.18 \\
8.66 \\
13.07 \\
1.84 \\
0.01 \\
0.06 \\
\end{array}$ \\
\hline $\begin{array}{l}\mathrm{K}_{2} \mathrm{O} \\
\mathrm{P}_{2} \mathrm{O}_{5}\end{array}$ & $\overline{99.92}$ & $\overline{100.28}$ & $\overline{99.87}$ & $\overline{100.13}$ & 99.62 & $\overline{100.12}$ & $\overline{100.14}$ & $\overline{100.29}$ & $\overline{100.20}$ & $\overline{100.38}$ & $\overline{100.43}$ & $\overline{100.50}$ & 99.97 & $\overline{100.24}$ & $\overline{100.10}$ & $\overline{100.38}$ & $\overline{100.38}$ \\
\hline \multicolumn{18}{|c|}{ Trace elements (ppm) } \\
\hline $\begin{array}{l}\mathrm{Rb} \\
\mathrm{Sr}\end{array}$ & $\begin{array}{r}0.0 \\
45.2\end{array}$ & $\begin{array}{r}0.0 \\
49.8\end{array}$ & $\begin{array}{r}0.3 \\
54.3\end{array}$ & $\begin{array}{r}0.0 \\
60.7\end{array}$ & $\begin{array}{r}0.3 \\
66.4\end{array}$ & $\begin{array}{r}0.0 \\
57.9\end{array}$ & $\begin{array}{r}0.0 \\
50.0\end{array}$ & $\begin{array}{r}0.3 \\
48.3\end{array}$ & $\begin{array}{r}0.1 \\
62.2\end{array}$ & $\begin{array}{r}0.1 \\
66.4 \\
26.7\end{array}$ & $\begin{array}{r}0.0 \\
67.2 \\
24.1\end{array}$ & $\begin{array}{r}0.1 \\
67.4 \\
25.4\end{array}$ & $\begin{array}{r}0.0 \\
58.1\end{array}$ & $\begin{array}{r}0.0 \\
62.0 \\
25.7\end{array}$ & $\begin{array}{r}0.4 \\
49.6 \\
24.2\end{array}$ & $\begin{array}{r}0.3 \\
48.7 \\
24.3\end{array}$ & $\begin{array}{r}0.0 \\
52.8 \\
24.4\end{array}$ \\
\hline $\begin{array}{l}\mathrm{Y} \\
\mathrm{Ga}\end{array}$ & $\begin{array}{l}26.8 \\
16\end{array}$ & $\begin{array}{l}24.6 \\
15\end{array}$ & $\begin{array}{l}25.1 \\
16\end{array}$ & $\begin{array}{l}27.7 \\
17\end{array}$ & $\begin{array}{l}30.4 \\
18\end{array}$ & $\begin{array}{l}26.6 \\
15\end{array}$ & $\begin{array}{l}23.6 \\
16\end{array}$ & $\begin{array}{l}23.4 \\
16\end{array}$ & $\begin{array}{l}25.7 \\
16\end{array}$ & $\begin{array}{l}26.7 \\
16\end{array}$ & $\begin{array}{l}24.1 \\
17\end{array}$ & $\begin{array}{l}25.4 \\
17\end{array}$ & $\begin{array}{l}25.1 \\
16\end{array}$ & $\begin{array}{l}25.7 \\
16\end{array}$ & $\begin{array}{l}24.2 \\
16\end{array}$ & $\begin{array}{l}24.3 \\
16\end{array}$ & $\begin{array}{l}24.4 \\
15\end{array}$ \\
\hline $\mathrm{Nb}$ & 0.9 & 0.6 & 1.5 & 1.4 & 0.7 & 0.9 & 0.3 & 0.3 & 0.9 & 1.1 & 0.2 & 0.9 & 0.8 & 0.9 & 1.1 & 0.6 & 1.5 \\
\hline $\mathrm{Zr}$ & 49.3 & 43.8 & 45.4 & 56.1 & 64.4 & 54.6 & 42.3 & 42.5 & 52.1 & 53.8 & 48.9 & 52.7 & 48.5 & 52.3 & 46.4 & 45.1 & 45.1 \\
\hline $\mathrm{Zn}$ & 83.4 & 76.3 & 63.7 & 79.3 & 86.4 & 64.7 & 54.4 & 56.0 & 56.9 & 62.7 & 57.3 & 55.2 & 64.4 & 72.8 & 71.9 & 60.5 & 55.7 \\
\hline $\mathrm{Ni}$ & 84 & 98 & 92 & 75 & 55 & 77 & 94 & 97 & 89 & 86 & 83 & 91 & 106 & 84 & & 109 & 114 \\
\hline $\mathrm{Cr}$ & 175 & 215 & 209 & 103 & 26 & 116 & 244 & 232 & 222 & & & & & & & 327 & 348 \\
\hline $\mathrm{v}$ & 324 & 304 & 303 & 290 & 333 & 295 & 276 & 264 & 280 & 285 & 269 & 271 & 277 & 291 & 292 & 282 & 293 \\
\hline
\end{tabular}


Table 3. Statistics for Hole 504B basalts.

\begin{tabular}{|c|c|c|c|c|c|c|c|c|c|c|c|c|c|c|c|c|}
\hline & \multicolumn{4}{|c|}{ Legs 69 and 70 (aphyric only) } & \multicolumn{4}{|c|}{ Leg 83 (aphyric only) } & \multicolumn{4}{|c|}{ Leg 111 (aphyric only) } & \multicolumn{4}{|c|}{ Leg 111 (all) } \\
\hline & Average & $\begin{array}{l}\text { Standard } \\
\text { deviation }\end{array}$ & Maximum & Minimum & Average & $\begin{array}{l}\text { Standard } \\
\text { deviation }\end{array}$ & Maximum & Minimum & Average & $\begin{array}{l}\text { Standard } \\
\text { deviation }\end{array}$ & Maximum & Minimum & Average & $\begin{array}{l}\text { Standard } \\
\text { deviation }\end{array}$ & Maximum & Minimum \\
\hline \multicolumn{17}{|c|}{ Major elements (wt \%) } \\
\hline $\mathrm{SiO}_{2}$ & 50.3 & 0.3 & 50.7 & 49.8 & 50.1 & 0.5 & 50.8 & 48.8 & 50.3 & 0.6 & 51.1 & 48.9 & 50.3 & 0.5 & 51.1 & 48.9 \\
\hline $\mathrm{TiO}_{2}^{2}$ & 0.94 & 0.04 & 1.00 & 0.88 & 1.00 & 0.08 & 1.14 & 0.84 & 0.96 & 0.12 & 1.24 & 0.75 & 0.96 & 0.10 & 1.24 & 0.75 \\
\hline $\mathrm{Al}_{2} \mathrm{O}_{3}$ & 15.61 & 0.56 & 16.70 & 14.90 & 14.83 & 0.38 & 16.08 & 14.31 & 15.23 & 0.85 & 16.90 & 13.80 & 15.41 & 0.76 & 16.90 & 13.80 \\
\hline $\mathrm{Fe}_{2} \mathrm{O}_{3}{ }^{*}$ & 9.97 & 0.56 & 10.69 & 8.83 & 10.68 & 0.60 & 11.62 & 9.67 & 10.46 & 0.84 & 12.50 & 9.26 & 10.34 & 0.70 & 12.50 & 9.26 \\
\hline $\mathrm{MnO}$ & 0.18 & 0.02 & 0.23 & 0.16 & 0.21 & 0.02 & 0.26 & 0.17 & 0.18 & 0.01 & 0.21 & 0.15 & 0.17 & 0.01 & 0.21 & 0.15 \\
\hline $\mathrm{MgO}$ & 8.28 & 0.18 & 8.55 & 7.99 & 8.49 & 0.37 & 9.09 & 7.72 & 8.33 & 0.41 & 9.18 & 7.16 & 8.27 & 0.43 & 9.18 & 7.16 \\
\hline $\mathrm{CaO}$ & 12.68 & 0.24 & 12.98 & 12.17 & 12.86 & 0.25 & 13.18 & 12.25 & 12.72 & 0.40 & 13.17 & 11.70 & 12.76 & 0.37 & 13.34 & 11.70 \\
\hline $\mathrm{Na}_{2} \mathrm{O}$ & 1.99 & 0.13 & 2.20 & 1.80 & 1.99 & 0.19 & 2.44 & 1.68 & 1.90 & 0.16 & 2.22 & 1.59 & 1.90 & 0.23 & 2.51 & 1.09 \\
\hline $\mathrm{K}_{2} \mathrm{O}$ & 0.14 & 0.09 & 0.36 & 0.04 & 0.02 & 0.01 & 0.03 & 0.01 & 0.01 & 0.00 & 0.02 & 0.01 & 0.01 & 0.00 & 0.02 & 0.01 \\
\hline $\mathrm{P}_{2} \mathrm{O}_{5}$ & 0.08 & 0.01 & 0.10 & 0.06 & 0.09 & 0.01 & 0.11 & 0.08 & 0.06 & 0.01 & 0.08 & 0.05 & 0.06 & 0.01 & 0.08 & 0.05 \\
\hline \multicolumn{17}{|c|}{ Trace elements (ppm) } \\
\hline $\mathrm{Rb}$ & 2.2 & 2.0 & 7.3 & 0.4 & 0.5 & 0.1 & 0.9 & 0.4 & 0.1 & 0.1 & 0.4 & 0.0 & 0.1 & 0.1 & 0.4 & 0.0 \\
\hline $\mathrm{Sr}$ & 66.9 & 6.0 & 78.0 & 57.0 & 53.4 & 6.1 & 66.0 & 43.0 & 57.9 & 6.4 & 66.4 & 43.7 & 58.5 & 6.0 & 67.4 & 43.7 \\
\hline $\mathrm{Y}$ & 23.3 & 1.4 & 25.6 & 21.5 & 24.7 & 2.2 & 28.0 & 20.5 & 24.5 & 2.6 & 30.4 & 20.1 & 24.3 & 2.3 & 30.4 & 20.1 \\
\hline $\mathrm{Ga}$ & 16.0 & 0.6 & 16.8 & 15.2 & 15.4 & 0.5 & 16.4 & 14.5 & 15.8 & 0.9 & 18.4 & 14.1 & 15.7 & 0.8 & 18.4 & 14.1 \\
\hline $\mathrm{Nb}$ & 0.5 & 0.1 & 0.7 & 0.4 & 0.7 & 0.2 & 1.1 & 0.4 & 0.8 & 0.3 & 1.5 & 0.3 & 0.8 & 0.4 & 1.6 & 0.2 \\
\hline $\mathrm{Zr}$ & 47.5 & 3.0 & 53.0 & 43.0 & 50.6 & 5.5 & 59.0 & 38.0 & 48.3 & 5.9 & 64.4 & 38.4 & 47.9 & 5.2 & 64.4 & 38.4 \\
\hline $\mathrm{Zn}$ & 78.0 & 7.0 & 90.0 & 66.0 & 84.6 & 10.5 & 104.0 & 67.0 & 73.8 & 10.0 & 98.8 & 55.3 & 70.4 & 10.8 & 98.8 & 54.4 \\
\hline $\mathrm{Ni}$ & 106 & 19 & 144 & 76 & 93 & 7 & 102 & 76 & 104 & 28 & 164 & 55 & 104 & 24 & 164 & 55 \\
\hline $\mathrm{Cr}$ & 362 & 45 & 463 & 282 & 274 & 59 & 355 & 168 & 252 & 106 & 388 & 26 & 263 & 96 & 413 & 26 \\
\hline $\mathrm{v}$ & 279 & 28 & 347 & 231 & 292 & 24 & 326 & 245 & 271 & 36 & 345 & 213 & 269 & 30 & 345 & 213 \\
\hline $\mathrm{Mg}^{\prime}$-value & 0.647 & 0.012 & 0.671 & 0.628 & 0.636 & 0.021 & 0.666 & 0.594 & 0.637 & 0.029 & 0.686 & 0.558 & 0.638 & 0.024 & 0.686 & 0.558 \\
\hline $\mathrm{CaO} / \mathrm{Na}_{2} \mathrm{O}$ & 6.39 & 0.43 & 7.09 & 5.66 & 6.54 & 0.61 & 7.73 & 5.02 & 6.75 & 0.74 & 8.21 & 5.27 & 6.82 & 1.07 & 12.03 & 4.98 \\
\hline $\mathrm{Zr} / \mathrm{Y}$ & 2.04 & 0.09 & 2.18 & 1.90 & 2.06 & 0.26 & 2.84 & 1.60 & 1.97 & 0.10 & 2.12 & 1.78 & 1.97 & 0.09 & 2.13 & 1.78 \\
\hline
\end{tabular}




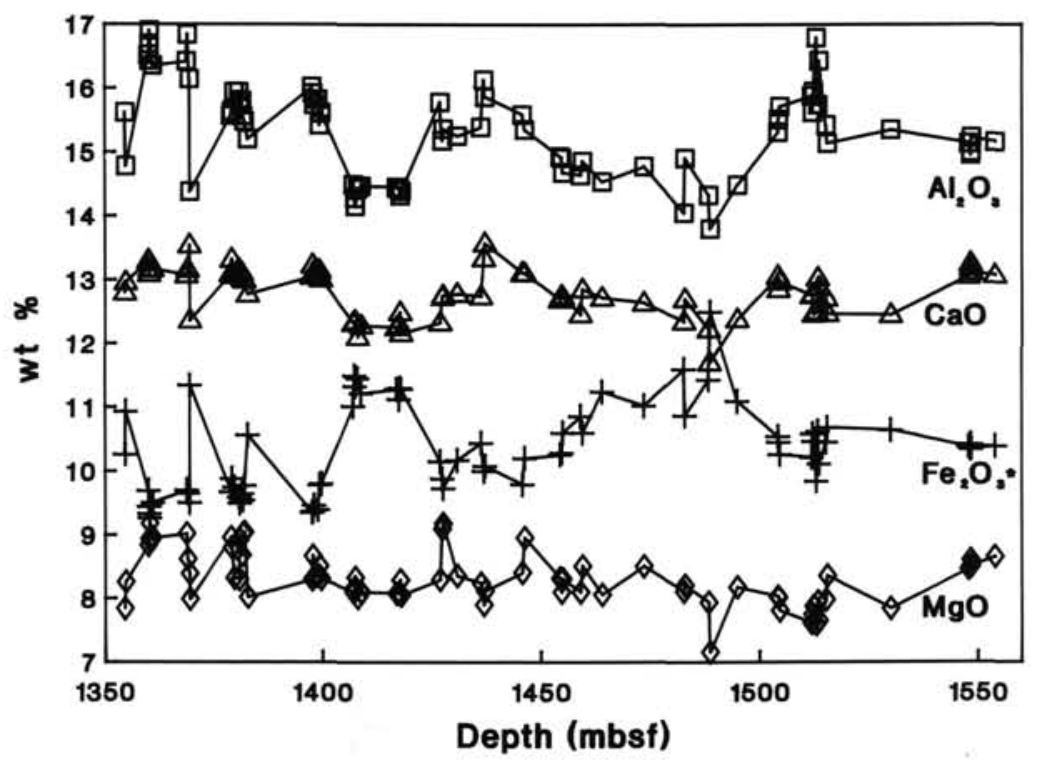

Figure 1. Major elements vs. depth for the Leg 111 basalts. The samples were analyzed by XRF (this study; Shipboard Scientific Party, 1988).

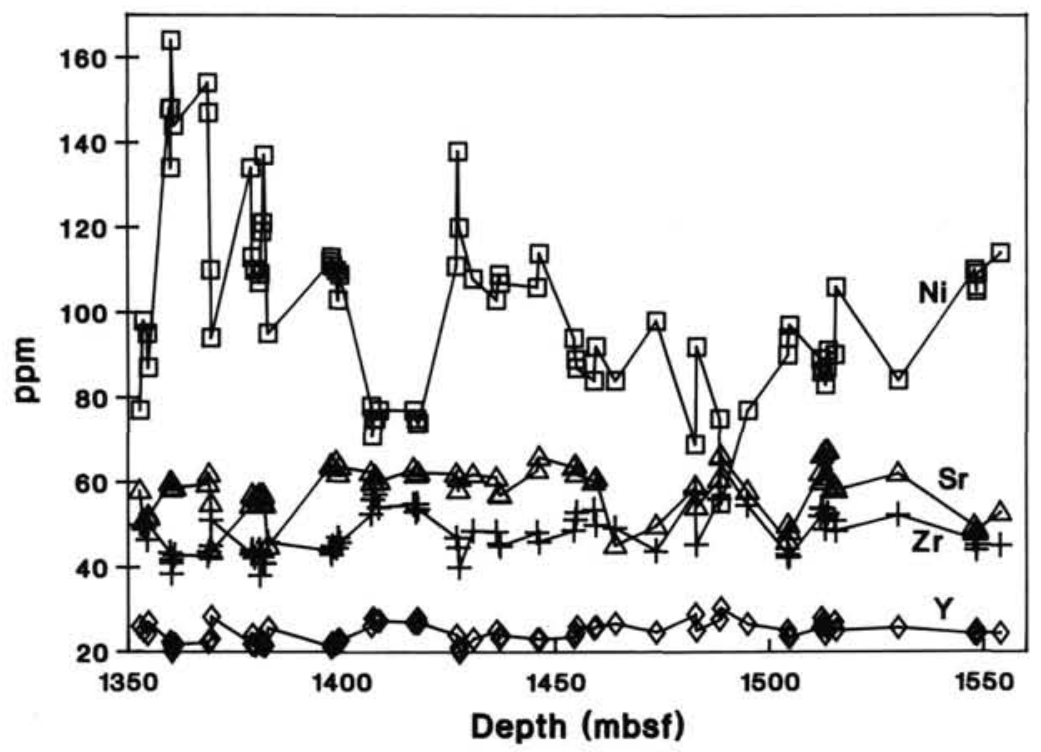

Figure 2. Trace elements vs. depth for the Leg 111 basalts. The samples were analyzed by XRF (this study; Shipboard Scientific Party, 1988).

ples crystallized olivine first, followed by olivine plus plagioclase, and finally olivine plus plagioclase plus clinopyroxene. Sample 111-504B-159R-1, 52-56 cm (Piece 9), lies at the extreme end of the Costa Rica Rift Zone trend $(\mathrm{Ni}=55 \mathrm{ppm}$ and $33 \%$ crystallization from the initial experimental composition) and has the most evolved trace element signature of the depleted basalts from Hole 504B.

$\mathrm{Zr} / \mathrm{Nb}$ ratios for the Hole 504B basalts recovered during Leg 111 are typical of Type I mid-ocean ridge basalts. As is the case for the Group D basalts from the upper part of Hole 504B (Autio and Rhodes, 1983; Kempton et al., 1985), Leg $111 \mathrm{Zr} /$ $\mathrm{Nb}$ ratios are greater than 30 (Fig. 7). Both $\mathrm{Nb}$ and $\mathrm{Zr}$ are highly depleted relative to other Type I mid-ocean ridge basalts at a similar stage of evolution (i.e., moderate abundances of compatible elements). Similarly, $\mathrm{Zr} / \mathrm{Y}$ ratios (1.8-2.1) are comparable to those observed in the depleted samples from the top of
Hole 504B. These values are unusually low in comparison with other mid-ocean ridge basalts (Fig. 8). Calculated fractionation paths for the two samples shown in Figure 6 (Autio, 1984) are given for reference in Figure 8. Again, Sample 111-504B-159R-1, $52-56 \mathrm{~cm}$ (Piece 9), lies at the far end of this trend although at a slightly higher $\mathrm{Zr} / \mathrm{Y}$ ratio than that of the experimental sample. The low ratios are similar to those found for $\mathrm{La} / \mathrm{Sm}\left(0.3-0.5_{\mathrm{CH}}\right.$; Autio and Rhodes, 1983; Etoubleau et al., 1983; Sharaskin et al., 1983; Kempton et al., 1985; Emmermann, 1985; Tual et al., 1985 ) and suggest a greater degree of depletion in highly incompatible elements $(\mathrm{Zr}, \mathrm{La}$, and $\mathrm{Ce})$ than in moderately incompatible elements (Y, Ti, and $\mathrm{Sm})$.

As seen in Figure 9, the $\mathrm{CaO} / \mathrm{Na}_{2} \mathrm{O}$ ratios $(5-8)$ are unusually high relative to those of other mid-ocean ridge basalts, whereas the $\mathrm{TiO}_{2}$ abundances are lower than normal. Calculated fractionation paths for single phases are shown for refer- 


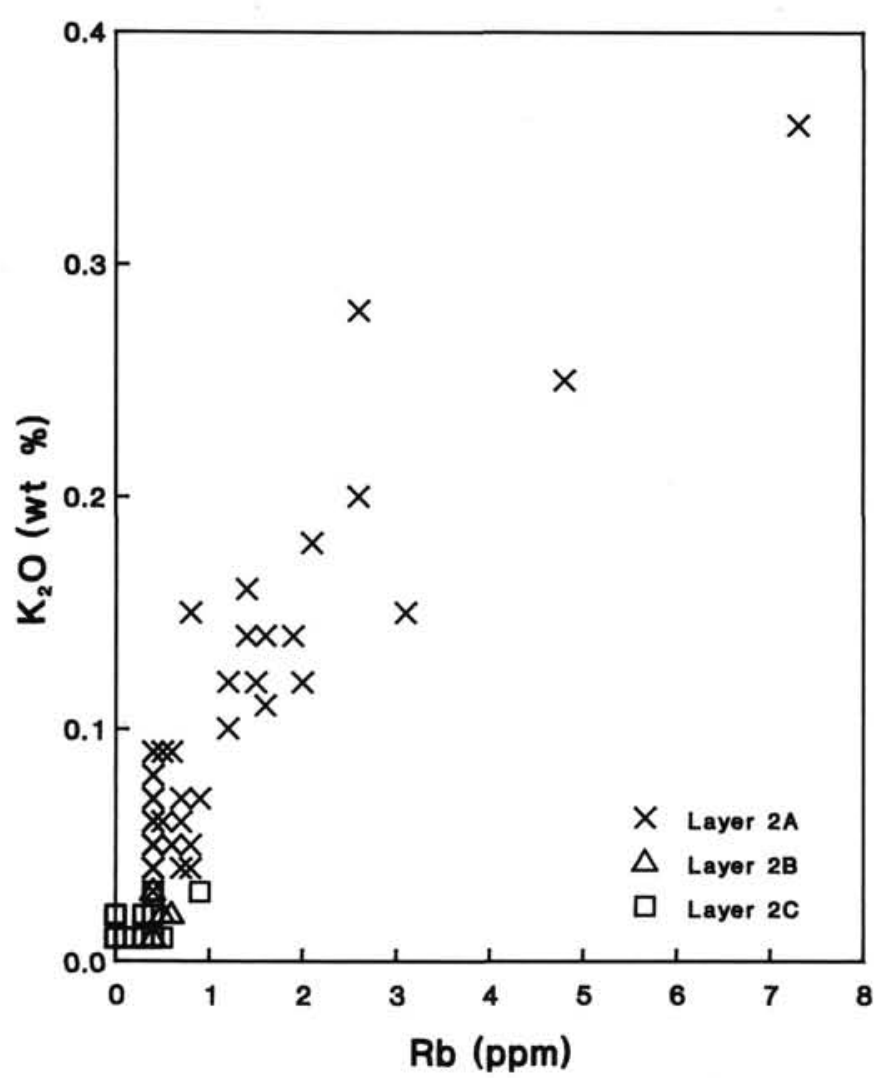

Figure 3. $\mathrm{K}_{2} \mathrm{O}$ vs. $\mathrm{Rb}$ as a function of depth. Only aphyric Group D samples are plotted. Analyses from Legs 69 and 70 (Layer $2 \mathrm{~A}$ ) are from Autio and Rhodes (1983), Leg 83 (Layers 2A, 2B, and 2C) from Kempton et al. (1985), and Leg 111 (Layer 2C) from this study. Both $\mathrm{K}_{2} \mathrm{O}$ and $\mathrm{Rb}$ vary strongly with depth as a function of the porosity of the samples and temperature of alteration (Seyfried and Bischoff, 1979), reaching maximums in the pillow lavas and decreasing in the transition and intrusive zones.

ence in Figure 9. Interestingly, the depleted Hole 504B samples plot at higher $\mathrm{Mg}^{\prime}$-values for a given $\mathrm{Ni}$ abundance (Fig. 10) than normal mid-ocean ridge basalts. This suggests that in addition to the depletion occurring in moderately incompatible elements (such as $\mathrm{Na}$ and $\mathrm{Fe}$ ), abundances of the compatible elements $\mathrm{Mg}$ and $\mathrm{Ca}$ have been raised in the mantle source.

\section{DISCUSSION}

The intrusive dike rocks of Layer $2 \mathrm{C}$, recovered on Leg 111, are virtually identical chemically to the depleted basaltic pillows and flows of Layer 2A from the upper part of Hole 504B. With the exception of the lack of recovery of the normal Type I and II basalts, which comprise approximately $2 \%$ of the Hole 504B samples from Legs 69,70 , and 83, the Leg 111 basalts are perfectly suitable to be considered the feeders for the extrusive units at the top of the hole. The slight differences in composition can be attributed to varying degrees of seawater alteration and/or phenocryst accurnulation.

While the Hole 504B basalts are unusually depleted in immobile magmaphile elements such as $\mathrm{Ti}, \mathrm{Zr}, \mathrm{Nb}, \mathrm{Y}, \mathrm{La}$, and $\mathrm{Sm}$ relative to other Type I mid-ocean ridge basalts (Figs. 7 and 8), compatible element abundances (e.g., $\mathrm{Mg}, \mathrm{Fe}, \mathrm{Ni}$, and $\mathrm{Cr}$; Figs. 5 and 6) are clearly much lower than, and inconsistent with, the values commonly taken to represent primitive basalts (Basaltic Volcanism Study Project, 1981). $\mathrm{Mg}^{\prime}$-values for the depleted Hole 504B basalts (Fig. 10) are higher than normal for the $\mathrm{Ni}$ abundances. Dependence on major element analyses alone may

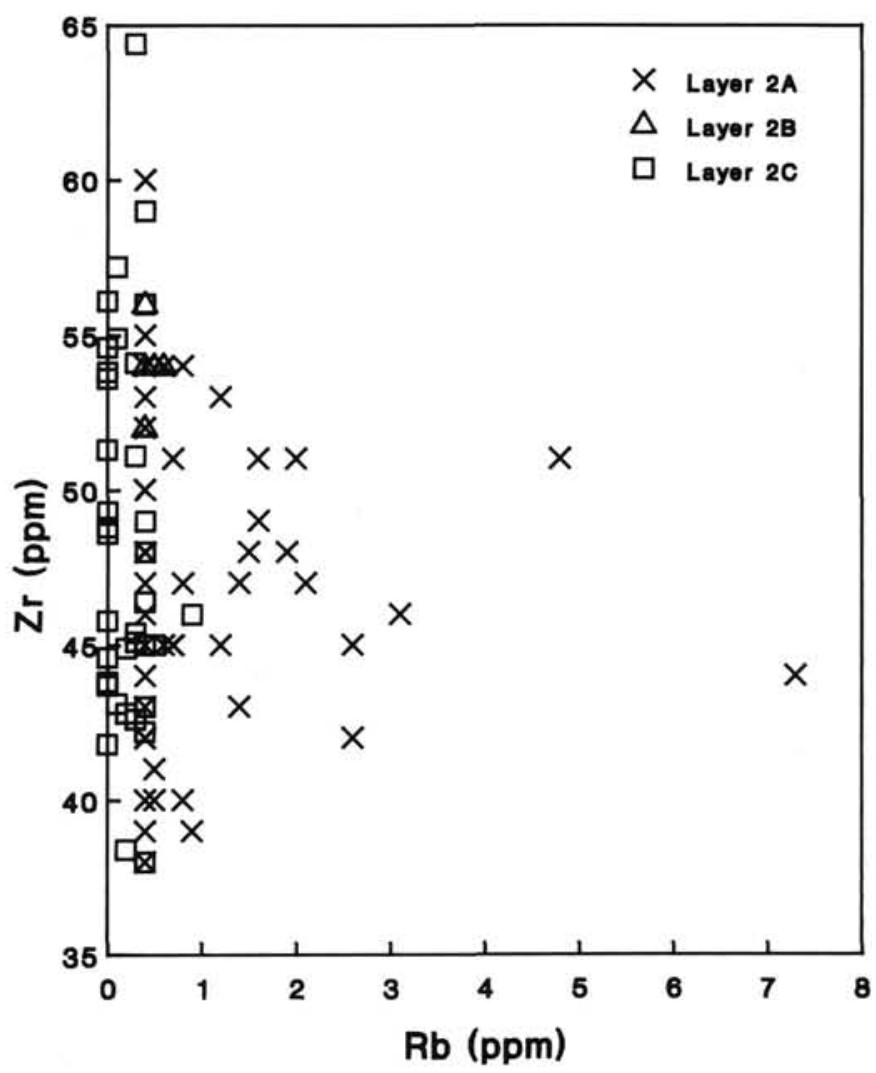

Figure 4. $\mathrm{Zr}$ vs. $\mathrm{Rb}$ as a function of depth. Only aphyric Group D samples are plotted. Analyses from the upper part of Hole 504B are from Autio and Rhodes (1983; Layer 2A) and Kempton et al. (1985; Layer 2B). In marked contrast to the behavior of $\mathrm{Rb}$ and $\mathrm{K}_{2} \mathrm{O}, \mathrm{Zr}$ abundances do not vary with depth. $\mathrm{Zr}, \mathrm{Ti}$, and $\mathrm{Y}$ have acted as relatively immobile elements and may be used to evaluate igneous processes.

have led to the misconception that these depleted basalts are primitive. Hole 504B basalts have been shown experimentally (Autio and Rhodes, 1983; Autio, 1984) to have olivine plus plagioclase, plus or minus clinopyroxene on the liquidus. Examination of the experimentally determined fractionation paths for the Hole 504B basalts (Figs. 6 and 8 ) shows that the depleted basalts follow these liquid lines of descent with some scatter. Sample 111-504B-159R-1, 52-56 cm (Piece 9), is the most evolved depleted sample recovered from Hole 504B, with extremely low abundances of compatible elements $(\mathrm{Ni}=55 \mathrm{ppm}$, $\mathrm{Cr}=26 \mathrm{ppm}$, and $\mathrm{Mg}^{\prime}$-value $\left.=0.56\right)$ and relatively high abundances of incompatible elements $\left(\mathrm{TiO}_{2}=1.24 \mathrm{wt} \%, \mathrm{Zr}=64\right.$ $\mathrm{ppm}$, and $\mathrm{Y}=30 \mathrm{ppm}$ ). From the ratios of highly incompatible immobile trace elements, it is estimated that $30 \%-40 \%$ fractionation is needed to go from the most primitive compositions observed to the composition of Sample 111-504B-159R-1, 52-56 $\mathrm{cm}$ (Piece 9). This estimate is supported by the similarity of the major element composition of Sample 111-504B-159R-1, 52-56 $\mathrm{cm}$ (Piece 9), to the experimental run product produced by $33 \%$ crystallization of one of the least evolved Costa Rica Rift Zone basalts (Autio, 1984). The congruence of estimates of percent fractionation based on major and trace element abundances suggests that major and trace elements have not been decoupled in this area.

It is impossible to fractionate the Costa Rica Rift Zone basalts to produce more normal mid-ocean ridge basalt compositions. The percentage of crystallization necessary to raise the levels of incompatible elements to more typical abundances would 


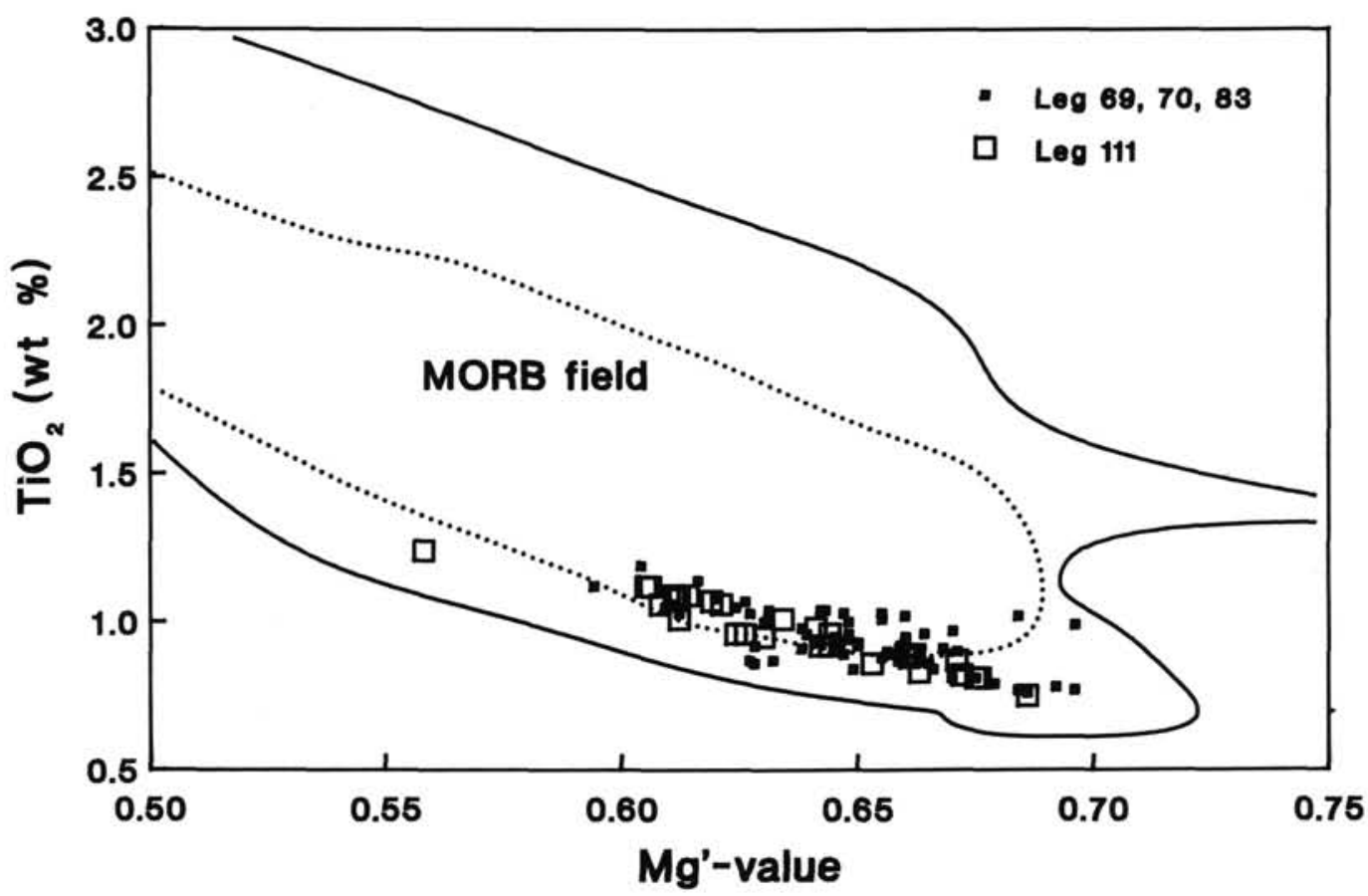

Figure 5. $\mathrm{Mg}^{\prime}$-value vs. $\mathrm{TiO}_{2}$. A large number $(>1300)$ of aphyric to sparsely phyric mid-ocean ridge basalt compositions were used to construct the MORB field. The dashed inner line delimits the range of $95 \%$ of these samples, and the solid line encloses the entire range. The aphyric Group D Hole 504B basalts (Autio and Rhodes, 1983; Kempton et al., 1985; this study) have $\mathrm{Mg}^{\prime}$-values lower than those of the magmas that have been considered candidates for primary melts (Frey et al., 1974; Basaltic Volcanism Study Project, 1981) and unusually low $\mathrm{TiO}_{2}$ contents for the $\mathrm{Mg}^{\prime}$-values.

produce compatible element concentrations much lower than those observed. It is evident from this data that extensive crystal fractionation of these melts produces lavas similar to Sample 111-504B-159R-1, 52-56 cm (Piece 9), and not normal mid-ocean ridge basalts (Figs. 6 and 8 ). Therefore, we conclude that the Hole 504B basalts are not primitive representatives of the midocean ridge basalt compositions, but rather, an unusually depleted type of moderately evolved ocean floor basalt. Other examples of this type of basalt composition have been found in the Indian Ocean (Melson et al., 1976) and as melt inclusions in phenocrysts within more normal mid-ocean ridge basalts (Donaldson and Brown, 1977; Dungan and Rhodes, 1978).

We have proposed elsewhere (Autio and Rhodes, 1981, 1983; Kempton et al., 1985) that one possible means of producing such depleted basalts at mid-ocean ridges is by multistage melting of a normal ocean ridge mantle source (Langmuir et al., 1977; Wood, 1979; Duncan and Green, 1980). Repeated melting of the source results in magmas that are highly depleted in magmaphile elements while retaining normal or slightly elevated abundances of compatible elements and normal isotopic (e.g., $\mathrm{Rb} / \mathrm{Sr}$ and $\mathrm{Nd} / \mathrm{Sm})$ and incompatible element $(\mathrm{Zr} / \mathrm{Nb}$ and $\mathrm{La} /$ Ce) ratios. Compositional data for the Leg 111 basalts are consistent with this model for the top section of Hole 504B, as it appears that the dikes feeding the overlying pillow and massive lavas are identical in composition.

Many glassy mid-ocean ridge basalts contain phenocrysts of plagioclase that are too calcic to be in equilibrium with their host glasses (Bryan et al., 1976; Rhodes et al., 1979; Basaltic Volcanism Study Project, 1981). These phenocrysts are not in equilibrium with even the most primitive compositions found on the ocean floor (Frey et al., 1974; Basaltic Volcanism Study Project, 1981). Multistage melting has been proposed as a method of producing the depleted liquids from which these an- orthite-rich phenocrysts were derived, followed by mixing with normal magmas in shallow axial magma reservoirs (Duncan and Green, 1980; Autio and Rhodes, 1983). The depleted Costa Rica Rift Zone basalts also contain highly calcic plagioclase phenocrysts (up to $\mathrm{An}_{90}$; Natland et al., 1983; Kempton et al., 1985; Kempton, 1985). However, unlike normal mid-ocean ridge basalts, the Hole 504B depleted basalts are calculated to be in equilibrium with unusually anorthitic plagioclase compositions $\left(\mathrm{An}_{85-92}\right.$; Autio and Rhodes, 1981, 1983) as a result of their high $\mathrm{CaO} / \mathrm{Na}_{2} \mathrm{O}$ ratios.

The Costa Rica Rift Zone basalts are the only samples described in detail that fulfill the compositional requirements of a second-stage melt. During the generation of mid-ocean ridge basalts, production of a small amount of depleted second-stage melt may be a common phenomenon. Subsequent mixing with earlier melts would obliterate all traces of the depleted melts except for their cognate phenocrysts. It is unclear why most of the rocks at the Costa Rica Rift retain their depleted characteristics. These samples do, however, provide us with a unique opportunity to view this component of the ocean floor much more directly.

Natland et al. (1983) have drawn attention to the "uniform" compositions of the Costa Rica Rift Zone basalts and have proposed that these are a product of a steady-state magma chamber. There are two problems with this theory. First, the depleted Hole 504B basalts are not especially uniform, although there are no systematic variations with depth. To derive the most evolved Group D compositions requires $30 \%-40 \%$ fractionation from the most depleted compositions. Second, the compositional variation in these basalts is too great to be solely the result of fractionation and mixing of a constant initial composition in a steady-state magma chamber, as defined by O'Hara (1977) and O'Hara and Mathews (1981). A fractionation path calculated 


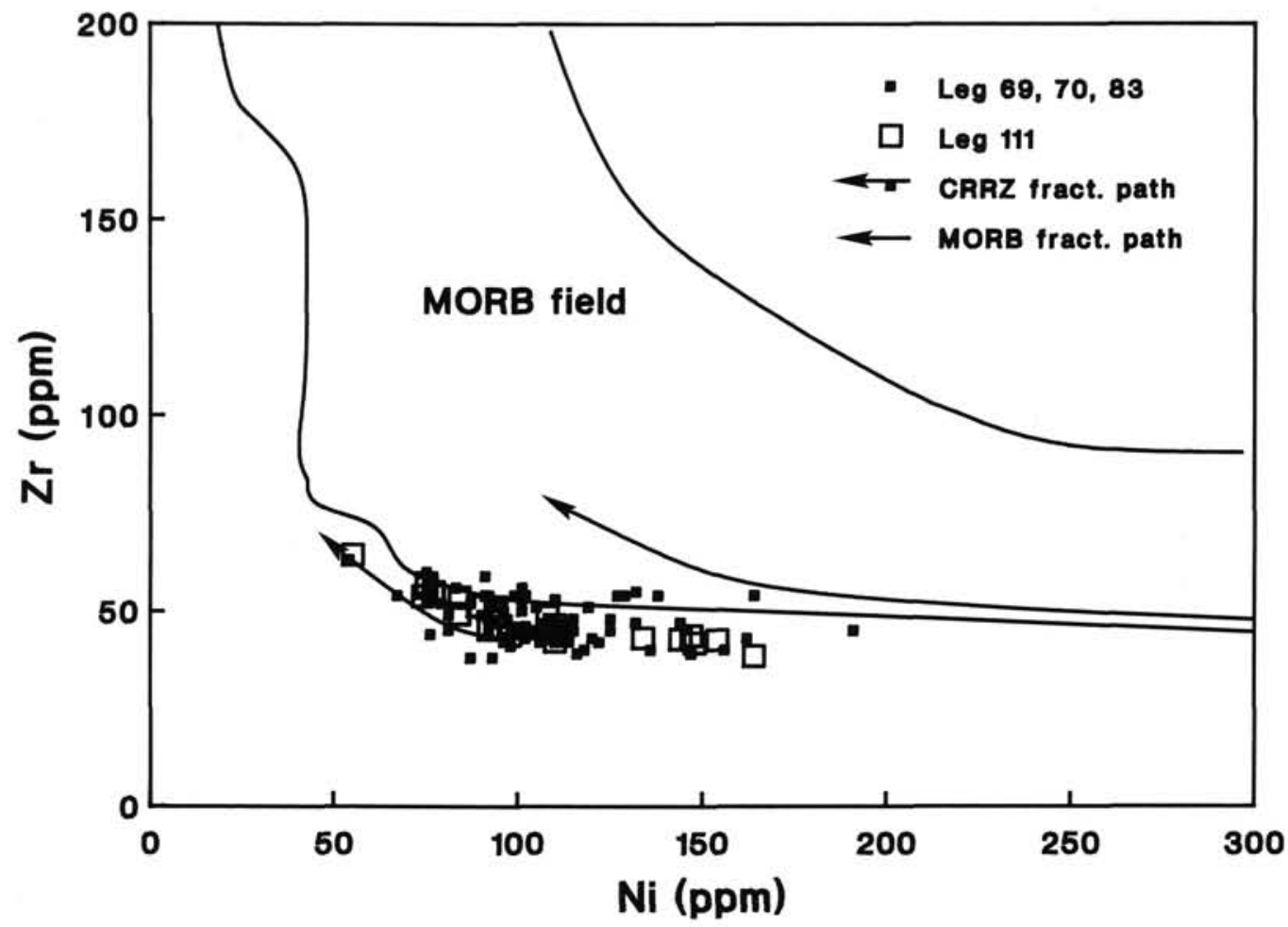

Figure 6. Ni vs. Zr. The MORB field was constructed as in Figure 5. Two liquid lines of descent calculated from 1atm experimental melting studies of ocean floor basalts (Autio, 1984) are shown for reference. The MORB fractionation path is for a typical primitive mid-ocean ridge basalt composition from Chain 43 on the Mid-Atlantic Ridge. The CRRZ fractionation path is for one of the least evolved Group D samples from the Costa Rica Rift Zone. Hole 504B basalts have lower abundances of $\mathrm{Ni}$ than proposed primary compositions despite their apparently primitive $\mathrm{Zr}$ abundances.

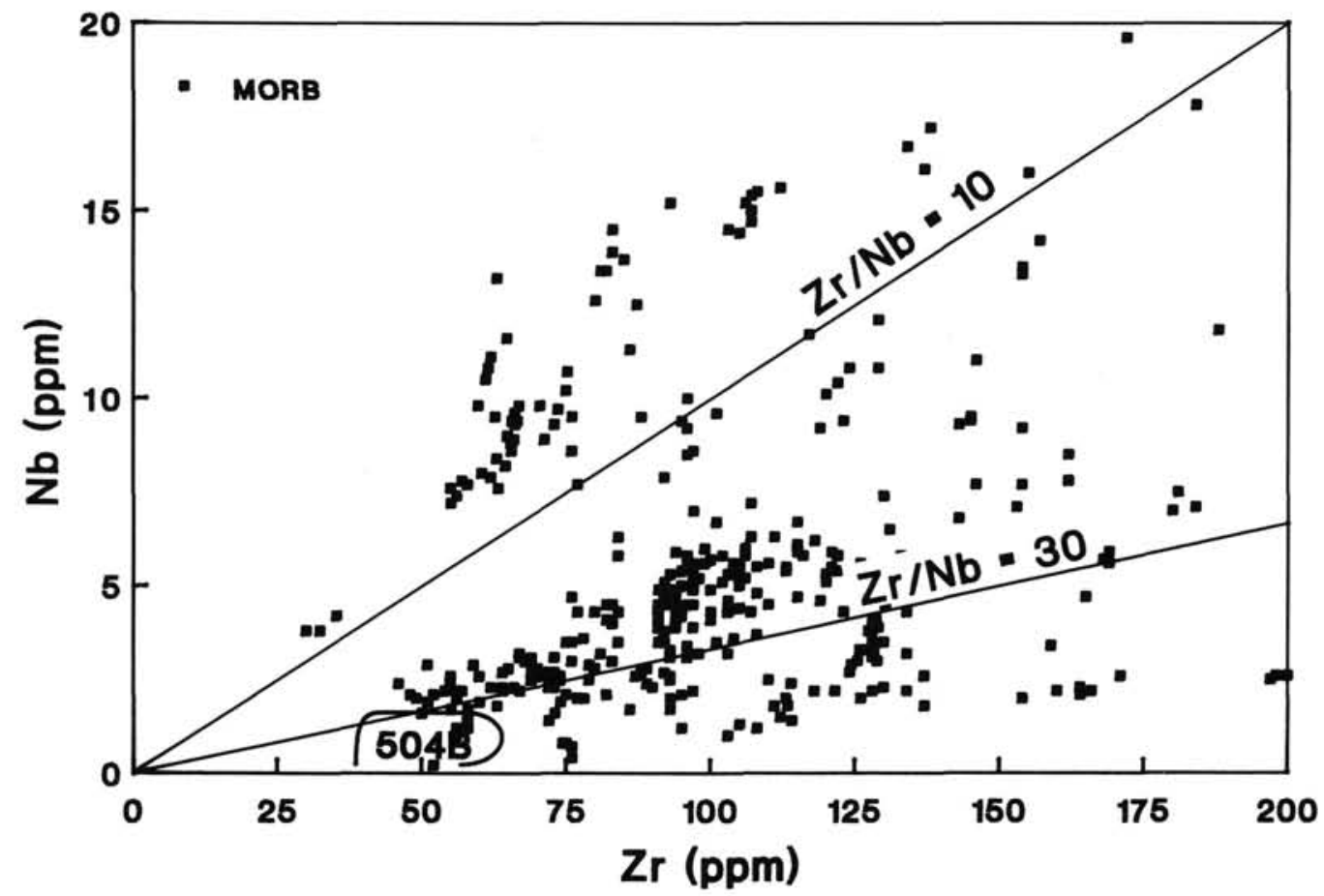

Figure 7. $\mathrm{Zr}$ vs. $\mathrm{Nb}$. Depleted Hole 504B basalts have ratios of $\mathrm{Zr} / \mathrm{Nb}$ similar to those of normal Type I midocean ridge basalts $(>30)$; however, abundances of both these elements are far below usual concentrations. 


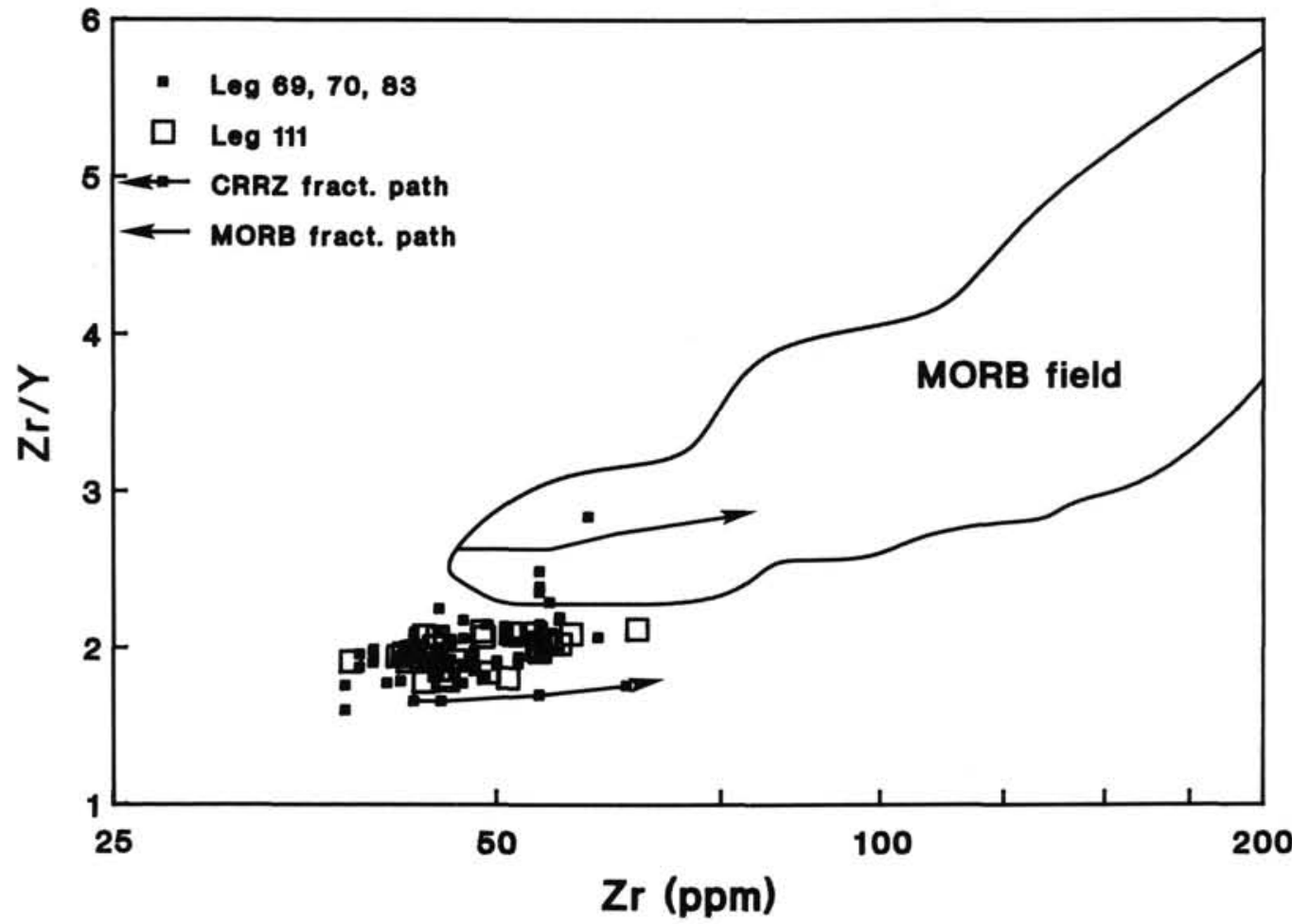

Figure 8. Zr/Y vs. Zr. Both Hole 504B aphyric Group D basalts and a MORB field for mid-ocean ridge basalts are plotted. Costa Rica Rift Zone basalts have lower than normal $\mathrm{Zr} / \mathrm{Y}$ ratios and $\mathrm{Zr}$ abundances. Only three published analyses have similar compositions. The two fractionation paths from Figure 6 calculated by Autio (1984) from experimental data are shown for reference.

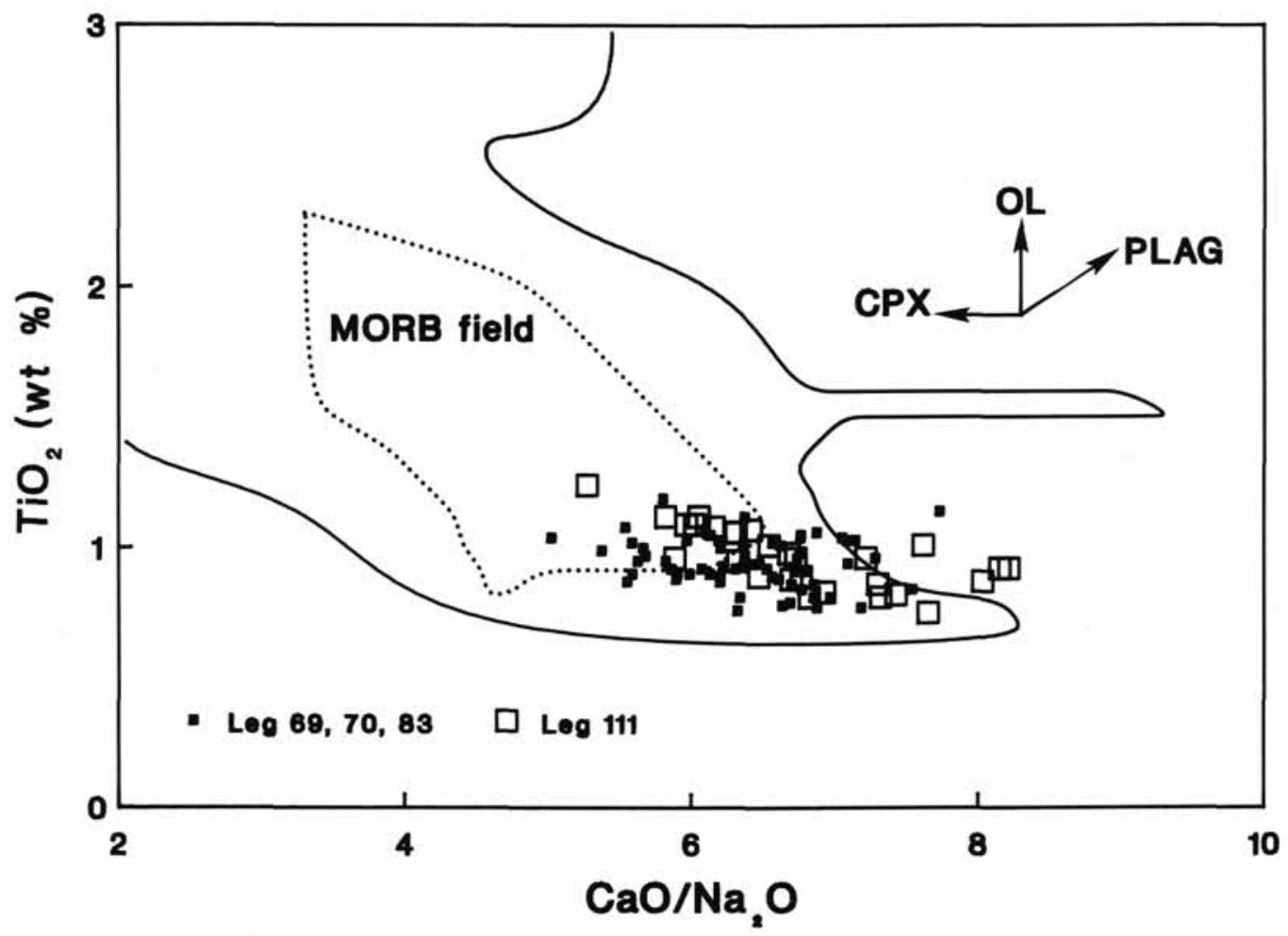

Figure 9. $\mathrm{CaO} / \mathrm{Na}_{2} \mathrm{O}$ vs. $\mathrm{TiO}_{2}$. Group $\mathrm{D}$ basalts from Hole 504B are shown with a field for mid-ocean ridge basalts (the inner line on the MORB field encloses $90 \%$ of the analyses). Depleted aphyric Hole 504B basalts have higher $\mathrm{CaO} / \mathrm{Na}_{2} \mathrm{O}$ ratios on average than the vast majority of mid-ocean ridge basalts. Vectors for the fractionation of olivine (OL), plagioclase (PLAG), and clinopyroxene (CPX) are shown for reference. 


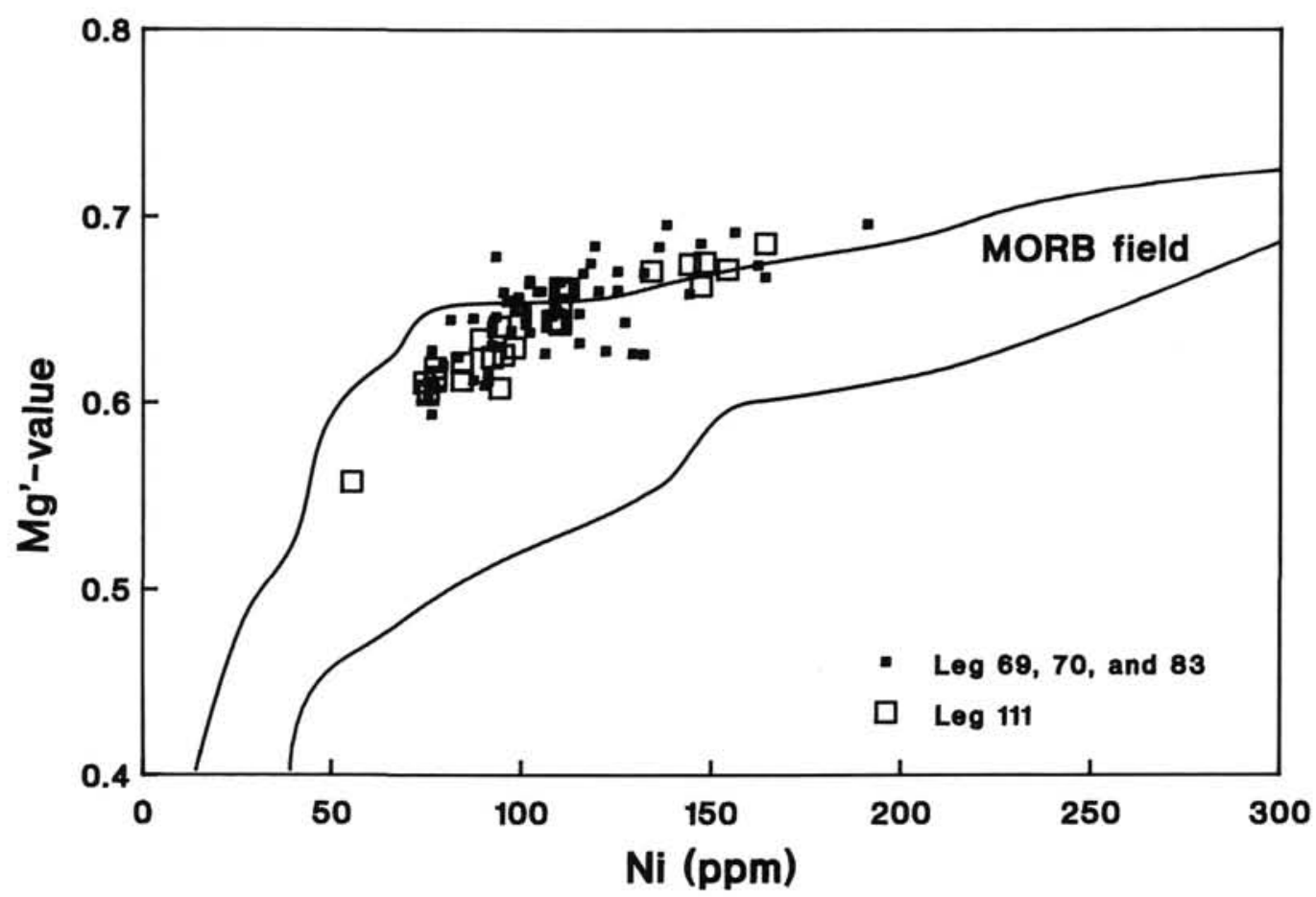

Figure 10. $\mathrm{Mg}^{\prime}$-value vs. Ni. The aphyric Group D Hole 504B basalts have higher $\mathrm{Mg}^{\prime}$-values for a given $\mathrm{Ni}$ content than most mid-ocean ridge basalts.

from experimental data (Autio, 1984) is shown for the plot of $\mathrm{Zr}$ vs. $\mathrm{Zr} / \mathrm{Y}$ in Figure 8 . Fractionation combined with mixing in primitive magmas of the same initial composition will move the composition of the resulting magma back and forth along the fractionation path. Error bars for the analyses are smaller than the scatter about the fractionation path, suggesting a third component. Repeated mixing of fractionating magmas derived by multistage melting of the source could produce the range of compositions observed.

\section{CONCLUSIONS}

Chemically, the Leg 111 basalts are virtually identical to the basalts recovered during Legs 69,70 , and 83 from the upper $1075 \mathrm{~m}$ of Hole 504B, with the exception of $\mathrm{Sr}, \mathrm{K}$, and $\mathrm{Rb}$ abundances. These elements have been affected to varying degrees by contact with seawater, depending on the porosity of the rock and temperature of interaction. Samples from the fractured and more porous pillow lavas of Legs 69 and 70 (the upper $571.5 \mathrm{~m}$ of Hole 504B) were altered at low temperatures $\left(<150^{\circ} \mathrm{C}\right.$; Seyfried and Bischoff, 1979; Honnorez et al., 1983) and are enriched in $\mathrm{Sr}, \mathrm{K}$, and $\mathrm{Rb}$ relative to the less porous transition zone from 571.5 to $780.5 \mathrm{~m}$ below seafloor (mbsf) (Leg 83) and dike zone from 780.5 to 1287.8 mbsf (Legs 83 and 111), which were affected by greenschist-level hydrothermal alteration (Seyfried and Bischoff, 1979; Alt et al., 1986; Shipboard Scientific Party, 1988). The dikes sampled on Legs 83 and 111 are the feeder system for the volcanic pillow lavas found in the upper part of the hole. The lack of systematic changes in igneous chemistry with depth suggests a long-term constancy of the processes (including repeated melting of the source, fractionation, and mixing) generating the Hole 504B basalts.

The Costa Rica Rift Zone basalts are highly depleted in magmaphile elements relative to other mid-ocean ridge basalts. Depletion in highly incompatible elements $(\mathrm{Nb}, \mathrm{Zr}$, and $\mathrm{La})$ is more extreme than the depletion in moderately incompatible elements
(Ti, Y, and $\mathrm{Sm}$ ), as seen in the unusually low $\mathrm{Zr} / \mathrm{Y}$ ratios (1.5-2.5) and La/Sm ratios (0.3-0.5; Kempton et al., 1985). Despite the unusually low concentrations of incompatible elements, the abundances of compatible elements are not correspondingly high. The levels of $\mathrm{Ni}, \mathrm{Cr}, \mathrm{Fe}$, and $\mathrm{Mg}$ correspond to those of moderately evolved normal Type I mid-ocean ridge basalts. The interpretation that the Hole 504B basalts are moderately evolved, based on compositional criteria, is in good agreement with petrographic studies of these basalts that found phenocrysts of olivine, oscillatory-zoned plagioclase, and clinopyroxene (Natland et al., 1983; Kempton et al., 1985; Kempton, 1985) and with 1atm melting experiments (Autio and Rhodes, 1983; Autio, 1984) that showed olivine and plagioclase plus or minus clinopyroxene on the liquidus. Thus, the low levels of incompatible elements do not indicate that the Costa Rica Rift Zone basalts are among the most primitive basalts in the mid-ocean ridge system nor that these basalts could produce typical mid-ocean ridge basalts upon further fractionation.

We concur with Autio and Rhodes (1983) that the most likely explanation for the depleted basalts of Hole 504B is generation by multistage melting from a normal Type I mid-ocean ridge basalt source. This explanation is consistent with the extremely low levels of magmaphile elements occurring in rocks that are clearly not primitive. Further work on the details of this hypothesis is in progress. The unusually high $\mathrm{CaO} / \mathrm{Na}_{2} \mathrm{O}$ ratios of the Costa Rica Rift Zone basalts have been found to be in equilibrium (Autio and Rhodes, 1981, 1983) with the anomalous highly anorthitic plagioclase commonly found in disequilibrium in many mid-ocean ridge basalt glasses (Bryan et al., 1976; Basaltic Volcanism Study Project, 1981). If the Hole 504B basalts are the product of multistage melting, then this suggests that multistage melting may be a small but pervasive component of ocean floor melting dynamics. Therefore, it is important to learn the details of the processes controlling the generation of the Costa Rica Rift Zone basalts in order to better understand the ocean ridge system as a whole. 


\section{ACKNOWLEDGMENTS}

This work was supported by a USSAC grant through the Texas A\&M Research Foundation to L. K. Autio, J. W. Sparks, and J. M. Rhodes. We would like to thank D. V. Hills for help with sample preparation and W. R. Autio for help with manuscript preparation. We also thank W. B. Bryan and R. A. Liias for generously sharing the unpublished data included in the mid-ocean ridge basalt fields.

\section{REFERENCES}

Alt, J. C., Honnorez, J., Laverne, C., and Emmermann, R., 1986. Hydrothermal alteration of a $1-\mathrm{km}$ section through the upper oceanic crust, Deep Sea Drilling Project 504B: mineralogy, chemistry, and evolution of seawater-basalt interactions. J. Geophys. Res., 91: $10,309-10,336$.

Anderson, R. N., Honnorez, J., Becker, K., Adamson, A. C., Alt, J. C., Emmermann, R., Kempton, P. D., Kinoshita, H., Laverne, C., Mottl, M. J., and Newmark, R. L., 1982. DSDP Hole 504B, the first reference section over $1 \mathrm{~km}$ through Layer 2 of the oceanic crust. Nature, 300:589-594.

Autio, L. K., 1984. Compositional diversity of mid-ocean ridge basalts: an experimental and geochemical study with emphasis on the depleted Costa Rica Rift Zone basalts [Ph.D. dissert.]. Univ. Mass., Amherst.

Autio, L. K., and Rhodes, J. M., 1981. Basalts from Holes 501 and 504B (Costa Rica Rift Zone): a possible source for high anorthite phenocrysts in ocean-floor basaltic magmas. EOS, Trans. Am. Geophys. Union, 62:309. (Abstract)

1983. Costa Rica Rift Zone basalts: geochemical and experimental data from a possible example of multistage melting. In Cann, J. R., Langseth, M. G., Honnorez, J., Von Herzen, R. P., White, S. M., et al., Init. Repts. DSDP, 69: Washington (U.S. Govt. Printing Office), 729-746.

Ballard, R., Bryan, W., Dick, H., Emery, K. O., Thompson, G., Uchupi, E., Davis, K. E., Boer, J., DeLong, S., Fox, P., Malcolm, F., Spydell, R., Stroup, J., Melson, W., and Wright, R., 1979. Geological and geophysical investigation of the Midcayman Rise Spreading Center: initial results and observations. In Talwani, M., Harrison, C. G., and Hayes, D. E. (Eds.), Deep Drilling Results in the Atlantic Ocean: Ocean Crust: Washington (Am. Geophys. Union), 66-93.

Barrett, T. J., 1983. Strontium and lead-isotope composition of some basalts from Deep Sea Drilling Project Hole 504B, Costa Rica Rift, Legs 69 and 70. In Cann, J. R., Langseth, M. G., Honnorez, J., Von Herzen, R. P., White, S. M., et al., Init. Repts. DSDP, 69: Washington (U.S. Govt. Printing Office), 643-650.

Barrett, T. J., and Friedrichsen, H., 1982. Strontium and oxygen isotopic composition of some basalts from Hole 504B, Costa Rica Rift, DSDP Legs 69 and 70. Earth Planet. Sci. Lett., 60:27-38.

Basaltic Volcanism Study Project, 1981. Basaltic Volcanism on the Terrestrial Planets: New York (Pergamon).

Bass, M. N., Moberly, R., Rhodes, J. M., Shih, C., and Church, S. E., 1973. Volcanic rocks cored in the central Pacific, Leg 17, Deep Sea Drilling Project. In Winterer, E. L., Ewing, J. I., et al., Init. Repts. DSDP, 17: Washington (U.S. Govt. Printing Office), 429-446.

Bender, J. F., Langmuir, C. H., and Hanson, G. N., 1984. Petrogenesis of basalt glasses from the Tamayo region, East Pacific Rise. J. Petrol., 24:213- 254.

Bryan, W. B., Thompson, G., Frey, F. A., and Dickey, J. S., 1976. Inferred geologic settings and differentiation in basalts from the DeepSea Drilling Project. J. Geophys. Res., 81:4285-4304.

Byerly, G. R., and Sinton, J. M., 1979. Compositional trends in natural basaltic glasses from Deep Sea Drilling Project Holes 417D and 418A. In Donnelly, T., Francheteau, J., Bryan, W., Robinson, P., Flower, M., Salisbury, M., et al., Init. Repts. DSDP, 51, 52, 53, Pt. 2: Washington (U.S. Govt. Printing Office), 957-972.

Christensen, N. I., and Salisbury, M. H., 1975. Structure and constitution of the lower oceanic crust. Rev. Geophys. Space Phys., 13:57-86.

Donaldson, C. H., and Brown, R. W., 1977. Refractory megacrysts and magnesium-rich melt inclusions within spinel in oceanic tholeiites: indicators of magma mixing and parental magma composition. Earth Planet. Sci. Lett., 37:81-89.

Drake, N.E.R., Rhodes, J. M., and Autio, L. K., 1985. The geochemistry of the Leg 82 basalts. In Bougault, H., Cande, S. C., et al., Init. Repts. DSDP, 82: Washington (U.S. Govt. Printing Office), 421-438.
Duncan, R. A., and Green, D. H., 1980. The role of multistage melting in the formation of oceanic crust. Geology, 8:22-26.

Dungan, M. A., and Rhodes, J. M., 1978. Residual glasses and melt inclusions in basalts from DSDP Legs 45 and 46: evidence for magma mixing. Contrib. Mineral. Petrol., 67:417-431.

Emmermann, R., 1985. Basement geochemistry, Hole 504B. In Anderson, R. N. Honnorez, J., Becker, K., et al., Init. Repts. DSDP, 83: Washington (U.S. Govt. Printing Office), 183-200.

Etoubleau, J., Corre, O., Joron, J. L., Bougault, H., and Treuil, M., 1983. Costa Rica Rift: variably depleted basalts in the same hole. In Cann, J. R., Langseth, M. G., Honnorez, J., Von Herzen, R. P., White, S. M., et al., Init. Repts. DSDP, 69: Washington (U.S. Govt. Printing Office), 765-774.

Fisk, M. R., Bence, A. E., and Schilling, J.-G., 1982. Major element chemistry of Galapagos Rift Zone magmas and their phenocrysts. Earth Planet. Sci. Lett., 61:171-189.

Frey, F. A., Bryan, W. B., and Thompson, G., 1974. Atlantic Ocean floor: geochemistry and petrology of basalts from Legs 2 and 3 of the Deep Sea Drilling Project. J. Geophys. Res., 79:5507-5527.

Friedrichsen, H., 1985. Strontium, oxygen, and hydrogen isotope studies on primary and secondary minerals in basalts from the Leg 83 section of DSDP Hole 504B, Costa Rica Rift. In Anderson, R. N., Honnorez, J., Becker, K., et al., Init. Repts. DSDP, 83: Washington (U.S. Govt. Printing Office), 289-296.

Honnorez, J., Laverne, C., Hubberton, H.-W., Emmermann, R., and Muehlenbachs, K., 1983. Alteration processes in Layer 2 basalts from Deep Sea Drilling Project Leg 70, Hole 504B, Costa Rica Rift. In Cann, J. R., Langseth, M. G., Honnorez, J., Von Herzen, R. P., White, S. M., et al., Init. Repts. DSDP, 69: Washington (U.S. Govt. Printing Office), 509-546.

Hubberton, H.-W., Emmermann, R., and Puchelt, H., 1983. Geochemistry of basalts from Costa Rica Rift Sites 504 and 505 (Deep Sea Drilling Project Legs 69 and 70). In Cann, J. R., Langseth, M. G., Honnorez, J., Von Herzen, R. P., White, S. M., et al., Init. Repts. $D S D P$, 69: Washington (U.S. Govt. Printing Office), 791-804.

Humphris, S. E., Bryan, W. B., Thompson, G., and Autio, L. K., in press. Morphology, geochemistry, and evolution of Serocki Volcano. In Detrick, R., Honnorez, J., Bryan, W. B., Juteau, T., et al., Proc. ODP, Sci. Results, 106/109: College Station, TX (Ocean Drilling Program).

Kempton, P. D., 1985. An interpretation of contrasting nucleation and growth histories from petrographic analysis of pillow and dike chilled margins, Hole 504B, Deep Sea Drilling Project, Leg 83. In Anderson, R. N., Honnorez, J., Becker, K., et al., Init. Repts. DSDP, 83: Washington (U.S. Govt. Printing Office), 165-181.

Kempton, P. D., Autio, L. K., Rhodes, J. M., Holdaway, M. J., Dungan, M. A., and Johnson, P., 1985. Petrology and geochemistry of basalts from Hole 504B. In Anderson, R. N., Honnorez, J., and Becker, K., et al. Init. Repts. DSDP, 83: Washington (U.S. Govt. Printing Office), 129-164.

Langmuir, C. H., Bender, J. F., Bence, A. E., and Hanson, G. N., 1977. Petrogenesis of basalts from the FAMOUS area: Mid-Atlantic Ridge. Earth Planet. Sci. Lett., 36:133-156.

LeRoex, A. P., Dick, H.J.B., Erlank, A. J., Reid, A. M., Frey, F. A., and Hart, S. R., 1983. Geochemistry, mineralogy, and petrogenesis of lavas erupted along the Southwest Indian Ridge between the Bouvet Triple Junction and $11^{\circ}$ east. J. Petrol., 24:267-318.

Marsh, N. G., Tarney, J., and Hendry, G. L., 1983. Trace element geochemistry of basalts from Hole 504B, Panama Basin, Deep Sea Drilling Project Legs 69 and 70. In Cann, J. R., Langseth, M. G., Honnorez, J., Von Herzen, R. P., White, S. M., et al., Init. Repts. $D S D P, 69$ : Washington (U.S. Govt. Printing Office), 747-764.

Melson, W. G., Vallier, T. L., Wright, T. L., Byerly, G., and Nelen, J., 1976. Chemical diversity of abyssal volcanic glass erupted along Pacific, Atlantic, and Indian Ocean sea-floor spreading centers. In Sutton, G. H., Manghnani, M. H., and Moberly, R. (Eds.), The Geophysics of the Pacific Ocean Basin and Its Margin: Washington (Am. Geophys. Union), 351-367.

Natland, J. H., Adamson, A. C., Laverne, C., Melson, W. G., and O'Hearn, T., 1983. A compositionally nearly steady state magma at the Costa Rica Rift: evidence from basalt glass and mineral data, DSDP Sites 501, 504, and 505. In Cann, J. R., Langseth, M. G., Honnorez, J., Von Herzen, R. P., White, S. M., et al., Init. Repts. $D S D P, 69$ : Washington (U.S. Govt. Printing Office), 811-858. 
Norrish, K., and Chappell, B. W., 1967. X-ray fluorescence spectrometry. In Zussman, J. (Ed.), Physical Methods in Determinative Mineralogy: New York (Academic Press), 161-214.

Norrish, K., and Hutton, J. T., 1969. An accurate X-ray spectrographic method for the analysis of a wide range of geological samples. Geochim. Cosmochim. Acta, 33:431-453.

O'Hara, M. J., 1977. Geochemical evolution during fractional crystallization of a periodically refilled magma chamber. Nature, 266:503507.

O'Hara, M. J., and Mathews, R. E., 1981. Geochemical evolution in an advancing, periodically replenished, periodically tapped, continuously fractionated magma chamber. J. Geol. Soc. London, 38:237-277.

Reynolds, R. C., 1967. Matrix corrections in trace element analysis by $\mathrm{X}$-ray fluorescence: estimation of the mass absorption coefficient by Compton scattering. Am. Mineral., 48:1133-1143.

Rhodes, J. M., Blanchard, D. P., Dungan, M. A., Rodgers, K. V., and Brannon, J. C., 1978. Chemistry of Leg 45 basalts. In Melson, W. G., Rabinowitz, P. D., et al., Init. Repts. DSDP, 45: Washington (U.S. Govt. Printing Office), 447-459.

Rhodes, J. M., Blanchard, D. P., Rodgers, K. V., Jacobs, J. W., and Brannon, J. C., 1976. Petrology and chemistry of basalts from the Nazca plate: part 2. Major and trace element chemistry. In Yeats, R. S., Hart, S. R., et al., Init. Repts. DSDP, 34: Washington (U.S. Govt. Printing Office), 239-244.

Rhodes, J. M., Dungan, M. A., Blanchard, D. P., and Long, P. E., 1979. Magma mixing at mid-ocean ridges: evidence from basalts drilled near $22^{\circ} \mathrm{N}$ on the Mid-Atlantic Ridge. Tectonophysics, 55: 35-62.

Schilling, J.-G., Zajac, M., Evans, R., Johnston, T., White, W., Devine, J. D., and Kingsley, R., 1983. Petrologic and geochemical variations along the Mid-Atlantic Ridge from $29^{\circ} \mathrm{N}$ to $73^{\circ} \mathrm{N}$. Am. J. Sci., 283: 510-586.
Seyfried, W. E., Jr., and Bischoff, J. L., 1979. Low temperature basalt alteration by seawater: an experimental study at $70^{\circ} \mathrm{C}$ and $150^{\circ} \mathrm{C}$. Geochim. Cosmochim. Acta, 43:1937-1947.

Sharaskin, A. Y., Migdisov, A. A., Rostschina, I. A., and Miklishansky, A. Z., 1983. Major and trace element chemistry of Hole 504B basalts and their alteration products (Costa Rica Rift, Deep Sea Drilling Project Leg 70). In Cann, J. R., Langseth, M. G., Honnorez, J., Von Herzen, R. P., White, S. M., et al., Init. Repts. DSDP, 69: Washington (U.S. Govt. Printing Office), 775-790.

Shipboard Scientific Party, 1988. Site 504: Costa Rica Rift. In Becker, K., Sakai, H., et al., Proc. ODP, Init. Repts., 111: College Station, TX (Ocean Drilling Program), 35-251.

Tarney, J., Saunders, A. D., Weaver, S. D., Donnellan, N.C.B., and Hendry, G. L., 1979. Minor-element geochemistry of basalts from Leg 49, North Atlantic Ocean. In Luyendyk, B. P., Cann, J. R., et al., Init. Repts. DSDP, 49: Washington (U.S. Govt. Printing Office), 657-691.

Tual, E., Jahn, B. M., Bougault, H., and Joron, J. L., 1985. Geochemistry of basalts from Hole 504B, Leg 83, Costa Rica Rift. In Anderson, R. N., Honnorez, J., Becker, K., et al., Init. Repts. DSDP, 83: Washington (U.S. Govt. Printing Office), 201-214.

Wood, D. A., 1979. Dynamic partial melting: its application to the petrogenesis of basalt lava series from Iceland, the Faeroe Islands, the Isle of Skye (Scotland) and the Troodos Massif (Cyprus). Geochim. Cosmochim. Acta, 43:1031-1046.

Date of initial receipt: 1 August 1988

Date of acceptance: 9 February 1989

Ms 111B-111 